\title{
Polymorphism of Garnet Solid Electrolytes and Its Implications on Grain Level Chemo-Mechanics
}

\author{
Marm B. Dixit ${ }^{* \dagger \ddagger}$ Bairav S. Vishugopi ${ }^{\S} \quad$ Wahid Zaman $^{\dagger}$ \\ Peter Kenesei $\quad$ Jun-Sang Park ${ }^{\ddagger}$ Jonathan Almer \\ Partha Mukherjee ${ }^{\dagger} \quad$ Kelsey B. Hatzell *ł‡\|
}

This manuscript has been authored by UT-Battelle, LLC, under Contract No. DEAC0500OR22725 with the U.S. Department of Energy. The United States Government retains and the publisher, by accepting the article for publication, acknowledges that the United States Government retains a non-exclusive, paid-up, irrevocable, world-wide license to publish or reproduce the published form of this manuscript, or allow others to do so, for the United States Government purposes. The Department of Energy will provide public access to these results of federally sponsored research in accordance with the DOE Public Access Plan (http://energy.gov/downloads/doe-public-access-plan).

${ }^{*}$ Lead Contact and Corresponding Author: kelsey.hatzell@princeton.edu and dixitmb@ornl.gov

${ }^{\dagger}$ Department of Mechanical Engineering, Vanderbilt University, Nashville, TN, 37240, USA

${ }^{\ddagger}$ Electrification and Energy Infrastructures Division, Oak Ridge National Laboratory, Oak Ridge, TN, 37830, USA

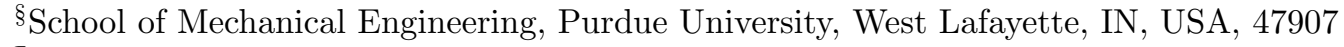

IX-ray Science Division, Argonne National Laboratory, 9700 South Cass Avenue, Lemont, Illinois 60439, USA

$\|$ Andlinger Center for Energy and Environment, Princeton University, Princeton, NJ, 08540, USA 


\begin{abstract}
Understanding and mitigating filament formation, short-circuit, and solid electrolyte fracture is a key necessity towards achieving practical SSBs. Herein, we employ a coupled far-field high energy diffraction microscopy -tomography approach for assessing chemo-mechanical behavior for dense, polycrystalline garnet $\left(\mathrm{Li}_{7} \mathrm{La}_{3} \mathrm{Zr}_{2} \mathrm{O}_{12}, \mathrm{LLZO}\right)$ solid electrolytes with grain-level resolution. Tracking the stress response for individual grains through in situ testing, failure onset and short-circuit mechanism is confirmed to be a stochastic, isolated process governed by the presence of local phase heterogenity. Coupling high energy X-ray diffraction and far field high energy diffraction microscopy measurements, these local regions are proposed to be regions with the presence of a cubic polymorph of LLZO arising potentially from local dopant concentration variation. Interplay of mechanics and transport is evaluated and coupled tomography and FF-HEDM dataset is employed to illustrate the degradation of polycrystalline garnet solid electrolyte. The results showcase pathways for processing high performing SSBs.
\end{abstract}




\section{Introduction}

Solid-state batteries (SSBs) offer the opportunity to leverage metallic anodes and high voltage cathodes to achieve safe, durable, and affordable secondary energy storage systems with high energy density ${ }^{1,2,3}$. However, intrinsic thermodynamic, electrochemical and mechanical instability between the components of the SSB significantly limits their performance ${ }^{4}$. These unfavorable electro-chemo-mechanical behavior at the numerous solid $\mid$ solid interfaces within the SSB leads to non-optimal material utilization, mechanical degradation, and poor ion transport ${ }^{1,5,6,7,8,9,10,11}$. These phenomena transcend several orders of magnitude in timeand length- scales and are yet to be fully understood. A fundamental understanding of interfacial design with regards to these physical processes by characterizing the structure and diagnosing the material responses are pivotal towards design of high-performance battery system in terms of driving range, safety, power, and durability ${ }^{12}$.

One key challenge with solid electrolytes (SEs) is their compatibility with Li metal anode. Achieving morphological control over repeated plating and stripping of Li metal is crucial for enabling durable SSBs ${ }^{12,13,14}$. Early modeling efforts by Monroe and Newman had proposed that high shear strength of solid electrolytes can potentially mitigate the development of unstable Li metal morphologies and prevent filament formation in batteries ${ }^{15,16}$. Extensive experimental results on a wide range of materials including garnets ${ }^{17}$, thiophosphates ${ }^{18}$, argyrodites ${ }^{19}$, anti-perovskites ${ }^{20}$, have conclusively shown the propensity of lithium to form filaments. In addition Several mechanisms are proposed for pathways for Li filament growth in solid electrolytes. These mechanisms can be distinguished by the nature of the anode $\mid \mathrm{SE}$ interface which can be (i) thermodynamically stable, (ii) unstable, and (iii) metastable based on the chemical makeup of the $\mathrm{SE}^{4}$. A key underlying mechanism for all these types of interfaces pertains to the limitations in mass transport of Li metal. High-rate electrodissolution of the anode can lead to formation of pores at the anode $\mid$ SE interface ${ }^{21,14}$. On a subsequent electrodeposition step, the pores act as magnifiers of electric field leading to localized, non-planar deposition resulting in filaments ${ }^{22,5}$. In addition to this, interphase formation in unstable anode $\mid$ SE interphases leads to large area specific resistances that cause strong electric and stress fields leading to the fracture of the solid electrolyte ${ }^{23,24}$. For metastable interfaces, microstructure-driven, Griffith-like cracking processes are also identified over and above the mass transport limitation mechanism ${ }^{22}$. Additionally, local deposition of Li metal due to trace electrical conductivities of material is also identified as a potential failure mechanism for the solid electrolytes ${ }^{12,13}$. Understanding the role and impact of heterogenities in the interface, interphase, and SE on filament formation and growth is vital. While a lot of work has been done on assessing these interplay for amorphous solid electrolytes (like $\left.\mathrm{Li}_{3} \mathrm{PS}_{6}\right)^{25,4,26,27,28,29}$, relatively much less work is carried out for polycrystalline solid electrolytes.

Garnet type $\left(\mathrm{A}_{3} \mathrm{~B}_{2}\left(\mathrm{XO}_{4}\right)_{3}\right)$ SEs possess high conductivity and electrochemical stability against Li-metal electrode ${ }^{30}$ and are one of the most promising SE material system. These ceramics have a Young's modulus of $>50 \mathrm{GPa}$ and shear modulus $\approx 60 \mathrm{GPa}$, which is significantly larger than the modulus of Li metal, suggesting a resilience against filament growth according to Monroe and Newman's model. A large series of garnet oxides have been developed as

$\mathrm{SE}$ of which one notable composition is $\mathrm{Li}_{7} \mathrm{La}_{3} \mathrm{Zr}_{2} \mathrm{O}_{12}(\mathrm{LLZO})^{31}$. This material exhibits two 
structures: a low temperature $\left(<700{ }^{\circ} \mathrm{C}\right)$ tetragonal symmetry with low ionic conductivity and a high temperature $(>700 \circ \mathrm{C})$ cubic structure which has a high ionic conductivity ${ }^{32}$. Aliovalent substitution of Ta into this matrix can help stabilize the cubic phase at low temperatures $^{33}$. Additional dopant systems are also investigated to maximize the ionic conductivities by tailoring the crystal structure of LLZO ${ }^{34}$. Recently, carefully designed experiments with LLZO have shown that the inherent reaction kinetics of Li deposition and dissolution at the LLZO | SE interface are extremely high and can sustain currents in the excess of $100 \mathrm{~mA} \mathrm{~cm}^{-2}$. However, most experimental studies on the meso-scale, report failure of the bulk SE below $1 \mathrm{~mA} \mathrm{~cm}^{-2}$. Mesoscale refers to the length scales at which homogenization breaks down and the material response to external stimuli (loading, field, etc.) is typically a result of an ensemble of individual constituents ${ }^{35}$. For the polycrystalline SEs, mesoscale refers to the ensemble of individual grains, distribution of voids and grain boundaries ${ }^{36,37}$. It is proposed that the presence of multitude of inter and intra-material interfaces within the polycrystalline solid electrolyte contribute to this accelerated failure ${ }^{38,39,40}$. The interplay between the individual grains, pores and grain boundaries and their intrinsic interfaces is not verified. Contradicting reports exist regarding the impact of grain size and grain boundaries on the failure of solid electrolytes. In general, failure modes in LLZO are typically linked to isolated deposition of $\mathrm{Li}$ in the bulk solid electrolyte as well as through filament propagation via the low-conducting, softer grain boundaries. Experimental investigation of precise failure modes of LLZO and the role of grain level chemo-mechanics is difficult due to the high density of the material and presence of high $\mathrm{Z}$ elements. Isolating and identifying the local failure onset and growth mechanism is crucial towards development of high performance solid-state batteries.

The key challenge with understanding the experimental behavior of these complex systems and developing effective chemo-mechanical material models is that each constituent demonstrates spatially anisotropic properties (eg. stiffness, conductivity, strength, among others) resulting from the local atomic configuration and structure ${ }^{41}$. These heterogeneous ensembles contribute to the resultant behavior like effective ionic conductivity, filament formation and fracture ${ }^{42}$. Mesoscale modelling efforts in solid-state batteries are typically focused on evaluating electro-chemo-mechanical phenomena with simplified domain approaches that do not capture the inherent stochasticity and complexity of the material systems ${ }^{43,44,45}$. So far, limited experiments have shown the ability to capture the full 3D microstructural features with grain-level resolution throughout an electrochemical testing process. Bulk-level, grain-resolved measurements of polycrystalline solid electrolytes allows to completely characterize the heterogeneities of stress distribution within the solid electrolyte and also enable detection of rare events such as void/crack nucleation. Synchrotron X-rays are the primary enabler for carrying out the experiments of the proposed complexity ${ }^{7}$. Specifically, spatially resolved measurements of microstructure and micromechanical states for mesoscale analysis are tomography and rotating crystal-method based diffraction measurements ${ }^{35}$. These techniques employ a high energy monochromatic X-ray that are imparted on a rotating sample while images of the diffracted beam in a transmission geometry are collected on an area detector over discrete angular intervals ${ }^{46,47,48}$. These techniques are referred to as high energy X-ray diffraction microscopy (HEDM). Far-field HEDM (FF-HEDM) technique provides a grain-resolved elastic strain tensor, the grain crystallographic orientation as well as 
the center-of-mass of each grain inside the probed polycrystalline sample. This makes it a perfect tool for investigation of polycrystalline garnet-type LLZO solid electrolytes that possesses an ensemble of randomly oriented grains (Fig. 1a). Mass transport between the grains is dependent on the orientation of the grains, grain boundary mismatch as well as the ion occupancy within the grains. During electrochemical operation, grains undergo chemomechanical strain from lithium ion motion as well as stresses generated from phenomena like lithium deposition and filament growth (Fig. 1a). Bulk ex situ mechanical measurements are routinely combined with imaging to rationalize chemo-mechanical material transformation $^{13,4,25,49,50,51}$. Yet, it is challenging to completely reconcile bulk mechanics with local effects brought on by material heterogenity (e.g. grain, defect, etc.). Far Field high energy diffraction microscopy (HEDM) is a non-destructive X-ray technique that provides the ability to measure full elastic strain tensor on a grain-by-grain basis in bulk samples ${ }^{52,53,54,55,56,57}$. In a typical FF-HEDM experiment, the sample is rotated from -180 to $180^{\circ}$ with respect to incoming X-rays at $0.1^{\circ}$ steps (Fig. 1b, S1-2). Diffraction images are captured on a 2D area detector at a large sample-detector distance. This diffraction images are processed to provide center of mass (COM), crystallographic orientations, sizes, and lattice strain tensor information.

In this work, we report in situ mesoscale microstructural characterization of LLZO garnet solid electrolytes by combined far-field high energy diffraction microscopy and x-ray tomography measurements. Far-field high energy diffraction microscopy enables a first-of-its-kind measurement of individual grains in a bulk polycrystalline solid electrolyte and provides stress growth in individual grains as a function of electrochemical cycling, while the tomography data-set captures the changes to the bulk microstructure of the solid electrolyte (porosity and cracking). The combined in situ characterization results in a correlated, multi-modal data set that enables quantification of a grain-resolved average strain tensor, structure and local crystallographic orientation of grains as well as allows for observations of microstruc-

tural features like voids/cracks. Grain tracking over multiple electrochemical cycling for a polycrystalline solid electrolyte allows for identifying the onset and growth of failure during symmetric cell cycling. Presence of trace secondary phases of LLZO are shown by FF-HEDM and high energy diffraction measurements. The interplay of structure-mechanics-transport are evaluated with experimental as well as mesoscale modeling studies (at the nm- to the umscale) provided a unified, correlated view of the evolving solid electrolyte under electrochemical cycling. This study is anticipated to inspire further research into these polycrystalline solid electrolytes with an emphasis on achieving local compositional and structural control.

\section{Results and Discussion}

\section{Chemo-Mechanical Response of Polycrystalline Solid Electrolyte}

Symmetric Li|LLZO|Li cells were cycled at the Advanced Photon Source in an custom-made cell holder (Fig. S3). The polarization profile is relatively flat during the first plating cycling indicating reversible Li electrodeposition in the system. The polarization profile begins to slope upward at high current densities which is consistent with void formation at the Li $\mid$ solid electrolyte interfaces ${ }^{28}$. Cycling lithium metal at high rates leads to filament formation and 
(a)

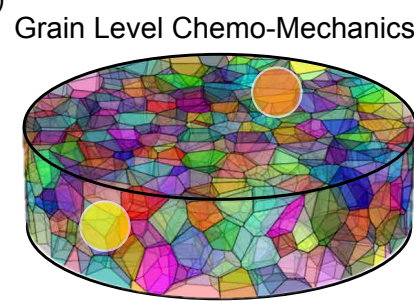

Polycrystalline Solid Electrolyte

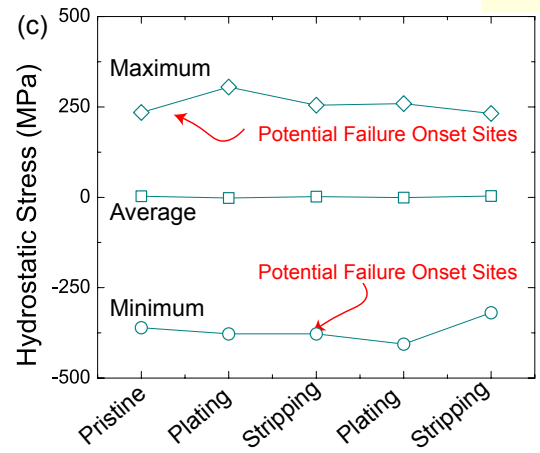

Unstrained

Neighborhood

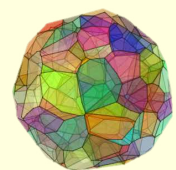

No Observable

Deformation

(d)
Strained Neighborhood

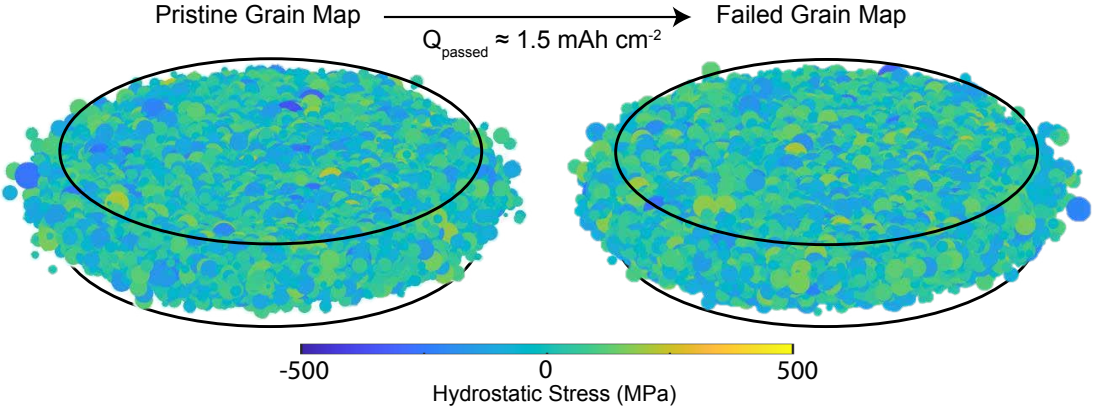

Figure 1: Mechanical Response of Poly-crystalline LLZO Material (a) Schematic diagram showing a polycrystalline pellet and associated phenomena with local strained and unstrained neighborhoods. The different colors in the first part of represent individual grains with differing orientations within the solid electrolyte. Under normal conditions, the local neighborhood comprises of different grain boundaries and voids and represents the "unstrained condition" for this study. Upon extended electrochemical cycling, local stoichiometric variations, Li deposition and crack formation can lead to mechanical stresses that alter the meso-scale structure of the solid electrolyte. (b) Schematic diagram showing the far-field high energy diffraction microscopy technique. The orange arrow impacting the sample from the left represents high energy monochromatic X-rays while the blue arrows emerging after the solid electrolyte is the diffracted X-ray beams. (c) Hydrostatic stress evolution during cycling of $\mathrm{Li}|\mathrm{LLZO}| \mathrm{Li}$ sample. The stress values are averaged over all the grains in the measured sample. Maximum and minimum values are shown for individual grains in the sample. Note that the red arrows are visual aid aimed to depict the minimum and maximum stress regions within the pellet as potential failure onset sites. (d) 3D grain maps for pristine and failed sample with the grains color mapped to the hydrostatic stress values. The two circles are drawn over the scatter plot to help in visualization of the solid electrolyte shape. A total of $1.5 \mathrm{mAh} \mathrm{cm}^{-2}$ lithium is cycled before failure.

electrical shorting. The garnet oxide solid electrolytes demonstrate a critical current density around $1.4 \mathrm{~mA} \mathrm{~cm}^{-2}\left(>5 \mathrm{MPa}, 50^{\circ} \mathrm{C}\right)$. Diffraction imaging experiments were conducted on cells with a characteristic diameter around $<2 \mathrm{~mm}$ in order to fit all components (electrode, electrolytes, etc.) within the field of view (Fig. S3). A total of $\approx 1.5 \mathrm{mAh} \mathrm{cm} \mathrm{cm}^{-2}$ charge is cycled through the system prior to failure of the in situ cell. In situ tomography and FFHEDM measurements were carried out on the pristine electrode and after each subsequent stripping and plating cycle. FF-HEDM provides the center of mass (COM), crystallographic orientation, and elastic strain of the individual grains in the sample illuminated by x-rays. While there are several strategies to determine these grain-resolved quantities ${ }^{58,59,60,61,62,63}$, in this work we use a previously developed MIDAS framework ${ }^{60,61,62}$. COM, orientation, and strain are determined assuming a cubic LLZO lattice with appropriate elastic constants. Approximately, 30,000 grains are identified with average grain sizes of $\approx 55-60 \mu \mathrm{m}$ which was consistent with tomography measurements. 
(a)

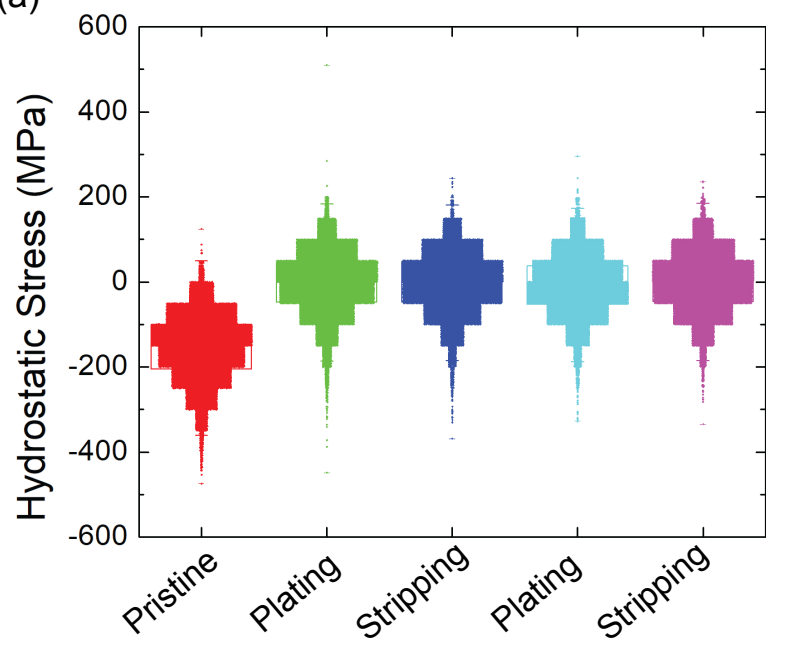

(b)

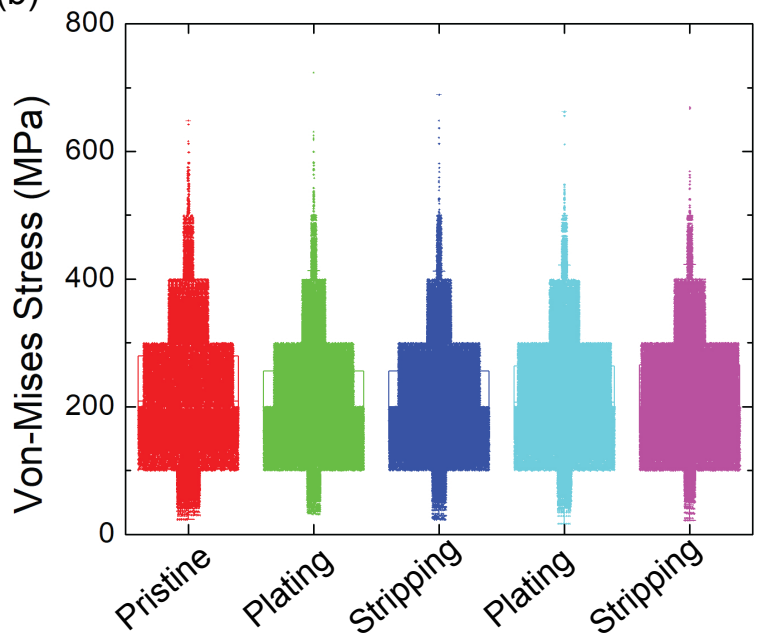

Figure 2: Stress Response of Poly-crystalline LLZO Material. Violin plots depicting the overall stress distribution along with the individual data sets for hydrostatic (a,c) stress and Von-Mises stress $(b, d)$. The width of the violin plots indicate the number density of grains at the specified stress value.

To simplify the analysis for this study, the hydrostatic and von-mises stress is utilized as a metric for comparison of the individual grains. FF-HEDM calculates the full strain tensor for individual grains via indexing. Corresponding stress tensor is calculated assuming a cubic lattice and employing the elastic modulus established for Al-doped LLZO obtained from first-principle calculations ${ }^{64}$. The hydrostatic stress is the average of the normal stresses acting on the grain while the von-mises stress provides an insight into the shear stresses acting on the material. Due to the underlying assumptions regarding the stress and strain distribution (isotropic), the absolute values are not employed directly, but the relative trends do provide insight into the grain-level chemo-mechanics of polycrystalline LLZO. The average hydrosatic stress value over the $\approx 30,000$ grains in LLZO at each cycling step shows negligible variation during cycling (Fig. 1c, S4). The minimum and maximum values among all the grains in the sample of the hydrostatic stress, however, show oscillating trends with lithium stripping and plating steps. These results were validated on an additional sample (Fig. S4). Similar profiles are also observed for average von-mises stress over the cycling stages (Fig. S5). The grain-specific stress distribution is visualized as violin plots (Fig. 2, S19). These plots represent the variability in stress (hydrostatic and von-mises) across the ensemble of grains (Fig. 2, S19). Highest width (most number of grains) for each electrochemical state is at similar stress levels consistent with the mean statistics (Fig. 1, S4). Lowest width (smallest number grains) are quantified at very high/low stress values. Thus, during cycling the majority of grains remain in a similar and uniform stress state and a minority of grains demonstrate significant deviations from the mean stress. This suggests that local microstructural heterogenity may exist within polycrystalline garnet oxides which results in individual grains that demonstrate heterogeneous physical properties.

There are various sources of 'local' microstructure heterogenity in a polycrystalline material including grains, grain boundaries, and defects. In addition, local heterogenity in 
structure (e.g. space group) may lead to non-uniform material properties within a polycrystalline solid electrolyte. The maximum and minimum stresses experienced by a minority of grains may be a result of a secondary phase. 3D Grain maps for pristine and failed sample show that the mechanical response in the sample is highly heterogeneous (Fig. 1d, S4). Additionally, full grain maps of the two measured samples over all the cycling steps show similar heterogeneity of stress distribution (Fig. S6).

\section{Evaluation of Secondary Phases in Bulk LLZO}

Indexing of the FF-HEDM data reveals that there are two cubic phases within the LLZO solid electrolyte: (a) a majority 220 space group (I-43d) and (b) minority 230 space group (Ia-3d) (Fig. 3e-f, S7). Both the space group showcase cubic symmetry, but have different Li- sites and co-ordinations. The stoichiometry of doping used in the current study indicates that the predominant structure would be $220 \mathrm{sg}$. I-43d as evidenced by the FF-HEDM and high resolution X-ray diffraction data (Fig. 1,4). Fitting the FF-HEDM data to 220 space group yielded approximately 30,000 grains, while fitting to the 230 space group only yielded 1000 grain fittings. To understand the implications of secondary phase on local ion transport and mechanical response of the solid electrolyte, mesoscale models based on kinetic Monte Carlo algorithm and finite element method (FEM) have been developed (see SI for detailed model description ${ }^{65,66}$. Distribution of ion occupancy within the bulk material that is embedded with a representative secondary phase is captured by the kinetic Monte Carlo model. Ion migration barriers was offset by $0.06 \mathrm{eV}$ between the two phases, in accordance to those reported in the experiments for the 220 and 230 structure. Uniform ion occupancy is maintained across the whole domain, after which the ion occupancy state dynamically evolves due to the difference in migration barriers between the two phases. For the simulations conducted here, the center region of the domain occupying a width of $7 \mathrm{~nm}$ is considered to be the secondary phase. Here, $i / i_{0}$ represents the occupancy descriptor, that has been normalized with respect to the initial ion occupancy $\left(i_{0}\right)$. An initial ion occupancy of $2 \%$ is assumed throughout the domain. Local occupancy of lithium ions are visualized in the domain after $4 \times 10^{8}$ migration events (Fig. 3b). Based on the results, we infer that ions preferentially migrate to the domain with the lower activation barrier with a significantly reduced occupancy in the domain with higher activation barrier. In the present context, 220 space group, which has the lower activation energy, forms the bulk of the pellet while the $230 \mathrm{sg}$. forms the secondary, isolated domains. This would result in local exclusion of ions and tortuous ion pathways around these secondary domains due to the inherent energy barriers associated with ion movement as suggested by the modeling results. To probe the mechanical stress and electric potential response, FEM simulations considering circular domains of $1 \mu \mathrm{m}$ of secondary phase embedded in the primary phase of $10 \times 10 \mu \mathrm{m}$ size are conducted. As measured in prior experiments, conductivity of the two phases were taken to be 0.3 and $1 \mathrm{mS} \mathrm{cm}^{-1}$, respectively. Since, limited data is available on the mechanical properties of these two cubic polymorphs of LLZO, a 3\% variation in the Young's modulus of the two phases was assumed in the mechanical simulations. While a larger variation may leads to quantitatively different stress profile, it is noted that the mechanical response trends in the vicinity of the secondary phase would still remain similar. For an externally applied 
(a)

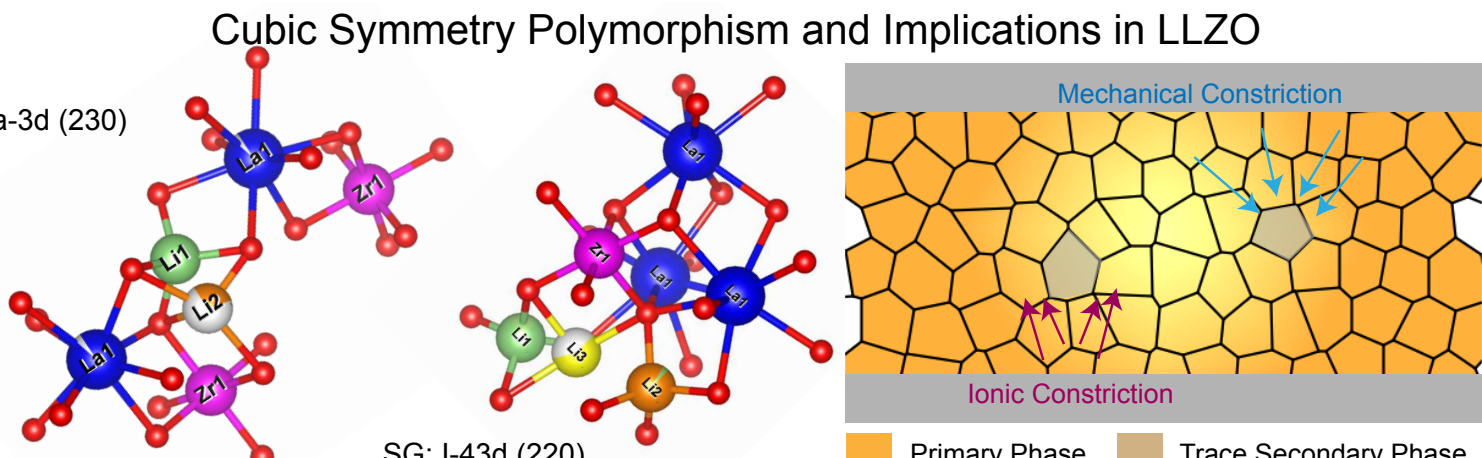

(b)

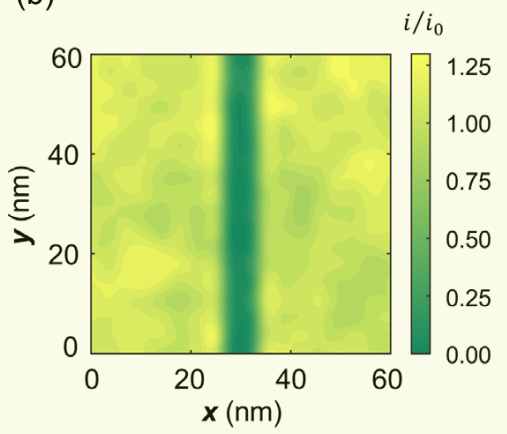

Local Li+ Occupation (c)

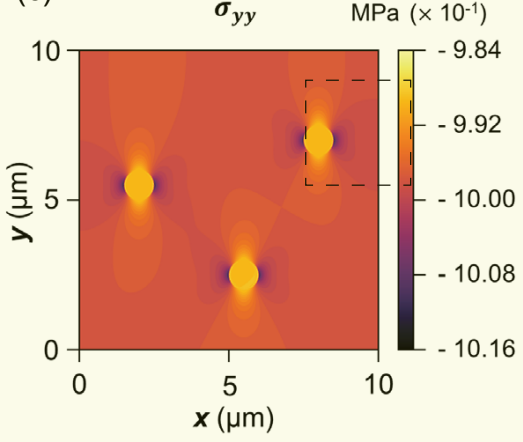

Mechanical Response (d)

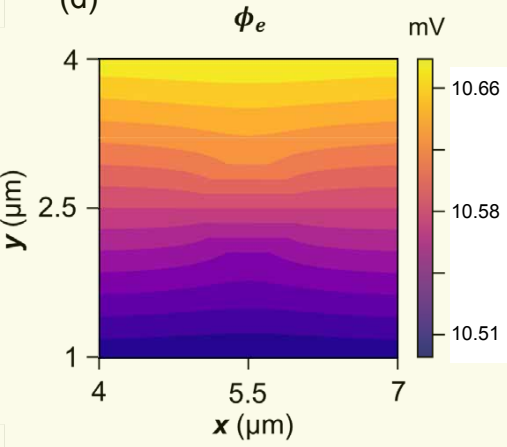

Electric Potential Response (e)

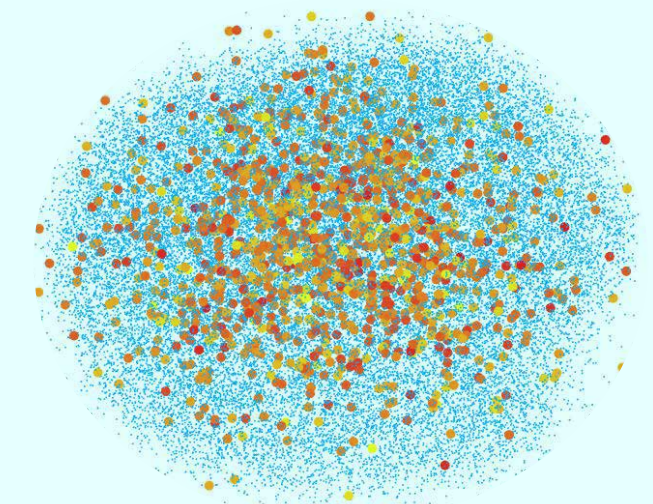

(f)

\section{Superposition of 220 SG grains (light blue) with 230 SG grains}

Figure 3: Local Phase anisotropy in Garnet Solid Electrolytes. (a) Lithium local environment in LLZO with the 220 and the 230 space groups. Schematic diagram highlighting the mechanical and ionic constriction effects in pellets due to the presence of a trace secondary phase. (b) Mesoscale modeling results highlighting the variation in local Li occupation due to presence of the secondary phase, with a differing ion migration barrier when compared to the bulk. (c) Mechanical and (d) Electric potential response of a domain with embedded secondary phases. For the local occupation and field response, the secondary phase is considered less conductive than the primary domain. (f) FF-HEDM dataset with neighborhood of high stress grains highlighted. An effective region of 70 $\mu \mathrm{m}$ is considered around each grain for plotting the neighborhoods. (g) Diffraction tomography results clearly showcasing the presence of trace secondary phases within the bulk of the pellet.

pressure of $1 \mathrm{MPa}$ and an applied current density of $0.5 \mathrm{~mA} \mathrm{~cm}^{-2}$, notable gradients in both stress and electric potentials are observed close to the secondary phase regions (Fig. 3c). 

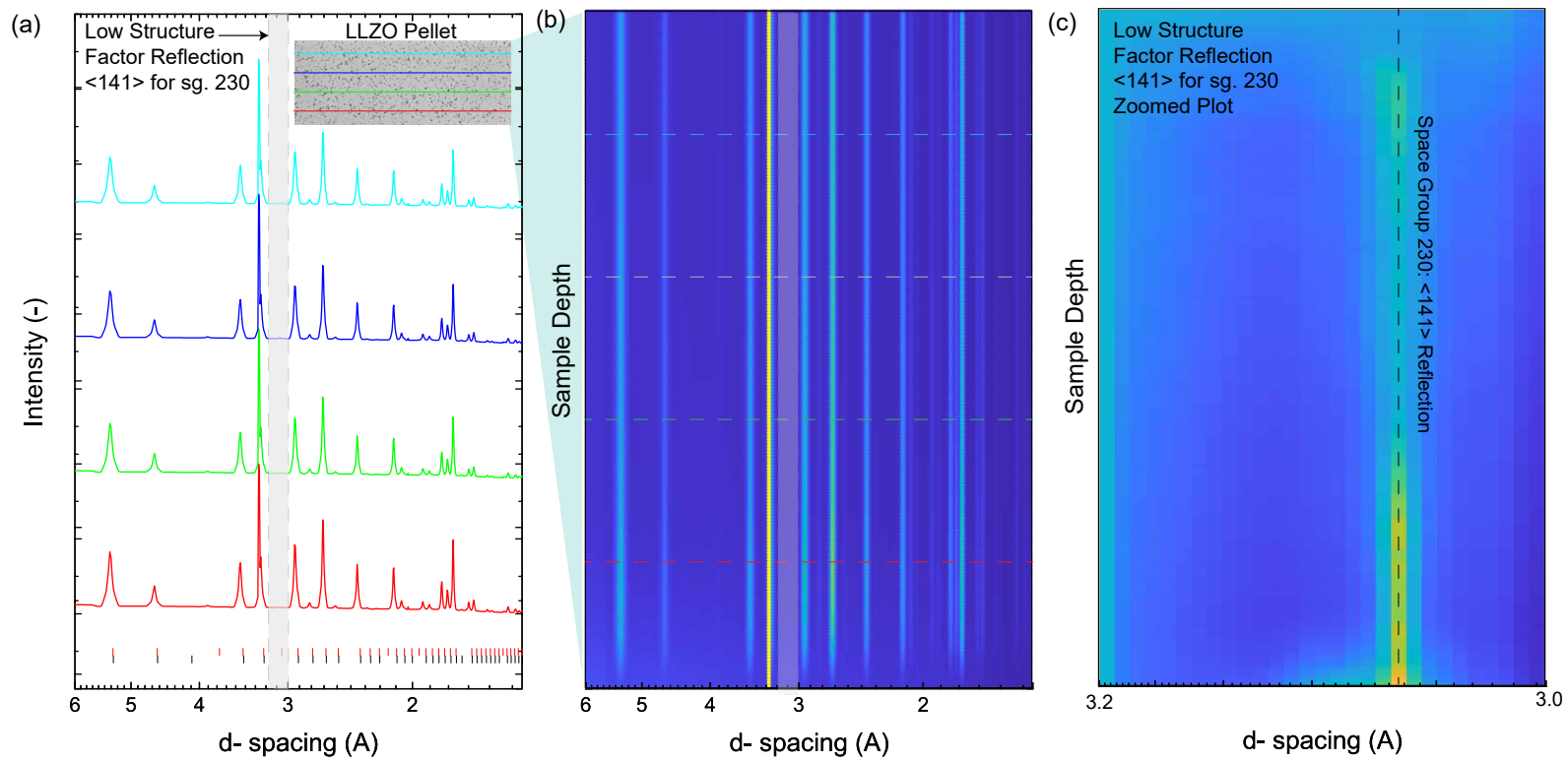

Figure 4: Evaluating Presence of Trace Phases in sintered LLZO Pellets. (a) High resolution, XRay diffraction patterns from specified locations of an LLZO pellet. (b) Spatially resolved high resolution XRD map of the LLZO pellet. (c) Spatially resolved XRD map zoomed to the anticipated location of the low-structure factor peak corresponding to $<141>$ plane in 230 space group LLZO.

Additionally, local occupation and field response are also evaluated for the case wherein the secondary phase is considered more conductive than the primary domain (Fig. S18). Even for this case, distinct occupation and field response in the vicinity of the secondary phase is observed. Isolated instances of the 230 space group within the bulk material are thus inferred to trigger the onset of heterogeneity in mechanical response and ion transport within the solid electrolyte pellet.

Earlier work for Ga-doped LLZO garnets showcased the presence of the two LLZO cubic polymorphs for LLZO with different doping concentrations ${ }^{67}$. The conventional cubic polymorph of LLZO, sg. 230 Ia-3d has a garnet structure with an eight-fold coordinated $\mathrm{La}^{3+}$ dodecahedra (24c), six-fold coordinated $\mathrm{Zr}^{4+}$ octahedra (16a) and $\mathrm{Li}^{+}$is located at interstitial sites showing tetrahedral (24d), octahedral (48g), and distorted four-fold (96h) coordination $^{68,69,70}$. For the $220 \mathrm{sg}$., lanthanum and zirconium occupy the same positions in the crystal structure. However, the local co-ordination of lithium and oxygen is different ${ }^{71}$. $\mathrm{Li}^{+}$ is found in three positions in I-43d, with two sites similar to that of the Ia-3d structure, and an additional partially occupied site on a general position 48e. This structure also showcases two independent $\mathrm{O}^{2-}$ positions in the general position again of $48 \mathrm{e}$. Prior experimental and modeling results have shown that the Ia-3d space group shows a higher activation energy $(\approx$ $0.31 \mathrm{eV})$ and lower conductivity $\left(\approx 0.2 \mathrm{mS} \mathrm{cm}^{-1}\right)$ compared to the I-43d structure $(\approx 0.25$ $\left.\mathrm{eV}, \approx 1 \mathrm{mS} \mathrm{cm}^{-1}\right)^{67}$. The improved transport properties for the I-43d structure result from a relatively flat energy landscape between the Li sites due to the presence of the neighboring supervalent cation. Local stoichiometry variation of the dopant element within the sintered pellets can lead to the formation a minority phase (230) in a predominantly 220 space group LLZO pellet. Theoretical reflections of 220 and 230 space group LLZO material shows that 
most reflections between the two space groups are common with a limited number of distinct reflections between the two space groups (Fig. S8). Additionally, the distinct reflections for the two space groups have very low structure factors that make detection between the space groups extremely difficult with traditional laboratory XRD. Local dopant element concentration variation can lead to isolated $230 \mathrm{sg}$. grains within the solid electrolyte (Fig. 4). This secondary phase results in local material heterogenity which can impact material properties (e.g. transport, electrochemical, mechanical, etc.) (Fig. 3a). Spatially resolved high resolution X-ray diffraction studies were carried out at the Advanced Photon Source (Fig. 4) to resolve the low structure factor peaks. A PILATUS detector was employed because it has a high dynamic range compared to the detector used for the FF-HEDM measurements. The higher dynamic range of the detector enables measurement of low intensity signals from the low structure factor reflections. A box beam was rastered vertically through the sintered LLZO pellet and the resultant diffraction patterns are visualized for individual layers, through the sample depth and for the region of the anticipated $<141>$ reflection for the 230 space group. The high resolution XRD maps show major reflections consistent with both space group polymorphs of LLZO (Fig. 4a,b). Additionally, the high dynamic range of the detector enables measurement of a unique $<141>$ reflection for the 230 space group (Fig. 4c). This reflection varies in intensity through the sample depth indicating a heterogeneous distribution of this phase within the sintered pellet. High resolution X-Ray Diffraction clearly showcases the presence of two polymorphs within a sintered pellet.

Based on combined evidence from the X-ray diffraction study and the modeling results, we expect the 230 space group to correspond with the local spikes in stress response inferred from (Fig. 1). While the local spikes in mechanics-driven signature were hypothesized to cause eventual failure of the solid electrolyte, here we identify that the 230 space group also leads to increase in ionic tortuosity and accumulation of Li-ions. Strong stress field gradients and preferential ion distribution in the 230 space group can potentially increase the severity of failure modes like cracking and isolated lithium deposition within the solid electrolyte, respectively. Additionally, from the FF-HEDM measurements during cycling, it was observed that the average grain stress does not show any variation. However, the minimum and maximum values showed oscillating trends with cycling (Fig. 1c, S4). It is proposed that these hot/cold-spots of stress reflect the presence of secondary phases, which is strongly supported by the high resolution X-ray diffraction measurements that confirm the presence of the secondary phase.

\section{Tracking Failure Mechanism Onset and Growth}

Several solid electrolyte failure onset and growth mechanisms are proposed in the literature ${ }^{21,22,13,12}$. These include growth and penetration of lithium dendrites, isolated deposition of lithium in bulk solid electrolyte, cracking and mechanical failure of solid electrolyte as well as unmitigated interphase growth ${ }^{72,26}$. In all these scenarios, the solid electrolyte is anticipated to undergo considerable chemo-mechanical deformation which would result in local strain variation. FF-HEDM dataset enables for the first time resolving an effective measure of the strain level in bulk pellet with grain-level resolution. Evolution of the strain tensor over all the grains in the bulk pellet is tracked to provide a definitive insight into the 

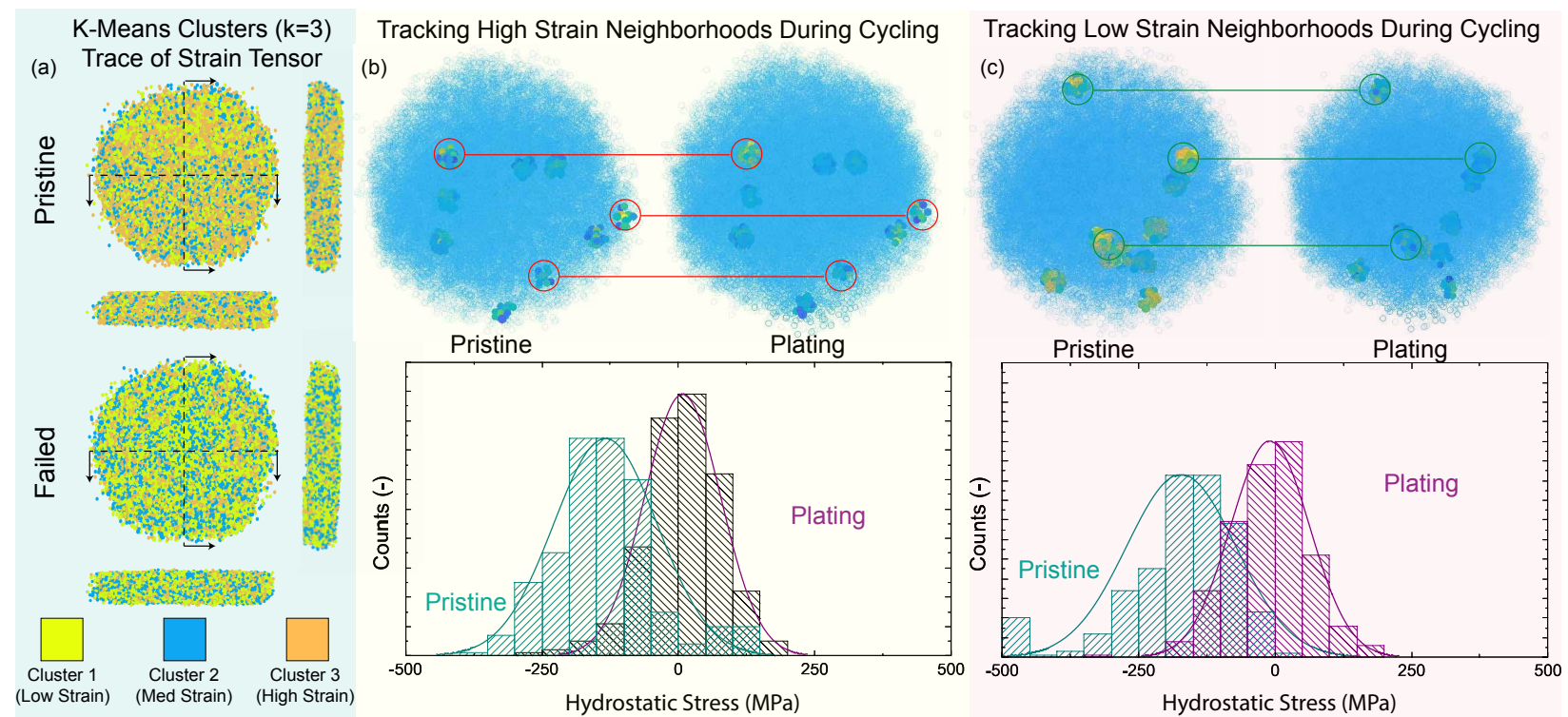

Figure 5: Hydrostatic Stress Evolution During Cycling. (a) Full pellet assessment for direction stress behavior using unsupervised machine learning algorithm, K-means. No preferential spatial distribution for the hydrostatic stress for individual grains is observed between the pristine and the failed sample. Tracking of (b) high stress and (c) low stress neighborhoods at two different stages of cycling, pristine and the first plating step along with the corresponding histograms and normal distribution fitting for the hydrostatic stress value for the grains in the identified neighborhood.

failure mechanism for the solid electrolyte. K-means algorithm was employed to identify if there is a directional behavior of strain growth within the bulk pellet. K-means algorithm is an unsupervised machine learning algorithm that segregates the input data into k- number of individual clusters based on the square errors between individual data points. The X, Y, $\mathrm{Z}$ position and hydrostatic stress for all the grains was fed to the k-means algorithm and three clusters predicted for pristine and the failed sample are visualized (Fig. 5a, S9). The results indicate no preferential direction of strain evolution between the pristine and the failed sample. Absence of bulk-scale trends in strain distributions indicate that failure of LLZO is driven by potentially a stochastic, isolated mechanism.

To further evaluate this hypothesis, local neighborhoods, in the vicinity of the strain hot/cold-spots are identified from the FF-HEDM datasets (Fig. 5b-c, S10). For the purpose of comparisons, ten grains with the highest and lowest values of hydrostatic stress are considered as hot-spots and cold-spots respectively. In order to effectively track between different cycling steps, a region of $2 \mathrm{x}$ the average size was assumed around the central hot-/cold-spot grain in order to define a neighborhood. Evolution of these neighborhoods during cycling is subsequently discussed out in terms of the hydrostatic stress of the grains located in these regions as well as corresponding microstructure variation (from the linked tomography dataset). The coupled FF-HEDM and tomography dataset presents a unique insight into the correlation of grain-level mechanical response of the LLZO material to the failure mechanisms during cycling. Tracking the low and the high strain neighborhoods from the pristine to the plating step (Fig. 5b-c), it is observed that the hydrostatic stress quantity in both hot- and cold- spot neighborhoods move to higher values after the first plating step. Ion transport through these polycrystalline materials is typically through hopping process 
(a)

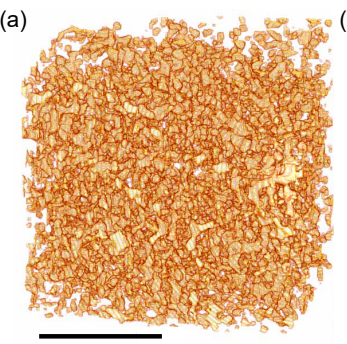

(b) Microstructure Transformation at Unstrained and Strain Hot-Spot (c)
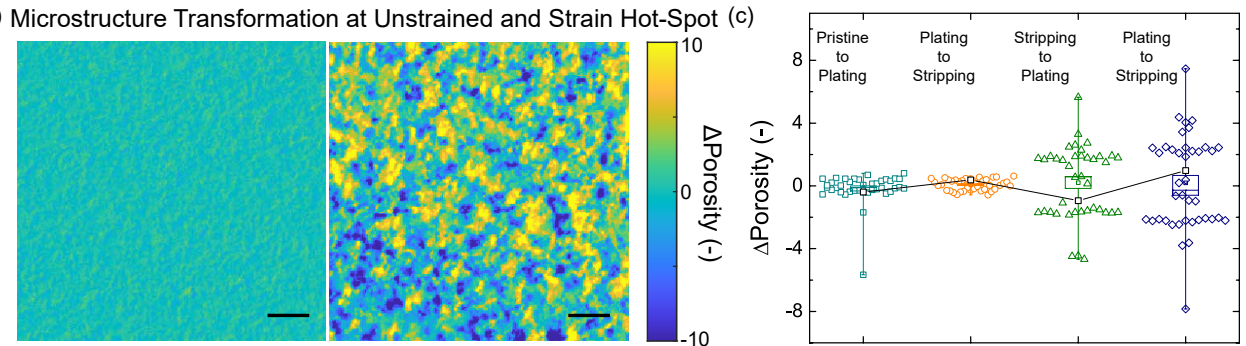

Figure 6: Correlating Grain-Level Mechanics to Bulk Microstructure. (a) Difference in the pore structure of a hot-spot neighborhood between the pristine and the failed sample. The structure represents the modification of pore network between pristine and the failed sample. The scale bar in the figure is $100 \mu \mathrm{m}$ (b) Depth averaged $\Delta$ porosity values for identical sub-volume sizes taken at a hot spot region (right) and other area (unstrained) region of the bulk pellet. The porosity difference is calculated between the pristine and the failed sample. The scale bar in the figure is 10 $\mu \mathrm{m}$. (c) Difference in porosity values tracked over the hot-spot and cold-spots for each step plotted as a box plot. Additionally, the average porosity difference over the entire pellet between each step is overlayed and connected by a solid line. As the sample undergoes degradation with cycling, the hot-/cold- neighborhoods clearly show very high variance in the porosity change compared to the bulk sample value.

where in the cation move through the solid electrolyte by hopping through available sites. As the transport mechanism is linked fundamentally through local stoichiometry and structure of the crystalline lattice, it is evident that ion transport through the system would lead to grain-level strain evolution. The consistent build up of stress from the pristine to the plating steps for both the hot/cold-spot strain neighborhoods suggest a transport driven mechanism whereby lattice distortion of the grains leads to higher strains for this step. As discussed earlier, the local hot-spots/cold-spots of strain are likely located at the secondary phases which act as ion transport bottlenecks with disparate ion occupancy at the interface as clearly showcased from the mesoscale simulations (Fig. 3c). In addition, the secondary phase would show disparate mechanical properties leading to stress accumulation. Subsequent tracking of the hot-/cold- neighborhoods through the cycling steps, further reaffirm this behavior (Fig. S11-S16). The evolution of hydrostatic stress values over the cycling step provides a clear validation of the hypothesis of secondary phase-driven degradation mechanisms like isolated Li deposition in garnet solid electrolytes.

\section{Correlation of Chemo-Mechanics and Bulk Microstructure}

To understand the interplay of grain-level chemo-mechanics to the microstructre, spatial locations corresponding to the hot/cold-spots identified by FF-HEDM data were identified in the tomography data-set that was collected simultaneously at each step. The grain maps and tomography data-sets were manually aligned for corrections for sample motion and rotations. Subsequently, sample subvolume at the regions of hot-/cold-spots of stress identified from the FF-HEDM dataset were extracted. Difference in microstructure at a hot-spot between the pore structure of the pristine and the failed sample is visualized (Fig. 6a). For this visualization, identical subvolumes of the hot-spot were cropped from the tomography dataset for the pristine and the failed sample. Subsequently, the binarized image stacks were 
algebraically subtracted to identify the difference image that is visualized here. The network represents the "change" in local microstructure between the pristine and the failed sample. It is evident that the local microstructure undergoes significant change upon failure at the hot-spot. Prior experimental studies have shown that changes in the X-ray transparent region (referred to as porosity) can arise from mechanical deformation (viz. cracks, chipping, among others) as well as can be a signature for local Li deposition within the bulk solid electrolyte. The mechanical and ionic constrictions that can occur at these hot-spots due to the secondary phase (Fig. 3), can lead to initiation of these multiple failure mechanisms and lead to the variation in microstructure that is observed at the hot-spot. Similar behavior is observed across multiple hot-/cold- spots evaluated for two distinct samples confirming these behavior (Fig. S9). To further illustrate this, the porosity difference values at a hotspot neighborhood and at another random un-strained location in the solid electrolyte is visualized (Fig. 6b, S9). The depth average porosity variation value between the pristine and the failed sample shows essentially no change in the local microstructure. However, at the region of hot-spot large structural variation is observed as illustrated by the depth-averaged porosity change values. Compiling the porosity change values at all the hot-/cold-spot neighborhoods for each cycle is subsequently tracked to understand the evolution of degradation through cycling. For the first plating and stripping cycle, no significant change is observed in the microstructure, as is also evident from the electrochemical data which shows flat polarization profiles and identical impedance values (Fig. S3). However, high variance in the local microstructure compared to the bulk porosity variation (solid line, in Fig. 6c), shows that the porosity changes at these hot-/cold- spot neighborhoods dominate the degradation of the solid electrolyte by either local Li deposition or cracking like phenomena. To further showcase the correlation between cell degradation and the local change in porosity, the standard deviation for the observed Li plating/stripping overpotential during each half-cycle and the standard deviation of the local porosity of the hot-spots identified are visualized (Fig. S20). Reversible lithium electro-deposition/dissolution is characterized by flat polarization profiles (low standard deviation) while filament growth leads to variations in the observed overpotentials (higher standard deviation). Strong correlation between the two data-sets are observed that suggest that the observed changes in local porosity play a dominant role in the degradation of the cell. Combined insight from the FF-HEDM and tomography clearly highlight the role of trace secondary phases on the degradation pathways of garnet solid electrolytes.

\section{Assessing Stress Induced Current Flows}

Another aspect that is of interest in describing the interplay of stress and failure mechanisms is the concept of stress-induced transport. Recent modeling results have shown that mechanical stresses can alter the energy landscape for deposition and can lead to conditions that promote localized electrodeposition ${ }^{6}$. Stress induced ion current is correlated to the molar volume of the cation, and the stress gradient of the system, with ions moving from regions of compressive stress to tensile stress. However, experimental validation of these mechanism is extremely difficult due to the relative difficulty in accessing spatially resolved stress field within bulk solid electrolytes. The stress identified by FF-HEDM measurements 
(a)

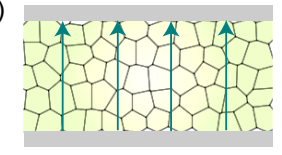

Direction 1:

Electrodeposition at Working Electrode

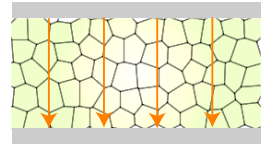

Direction 2:

Electrodissolution

(d) (b)

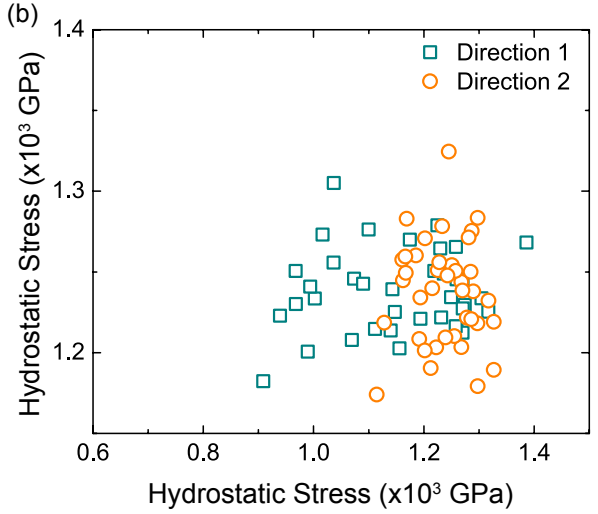

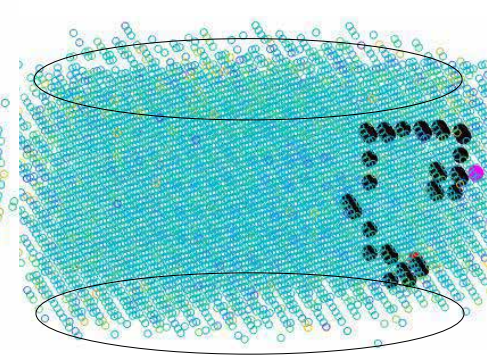

Strain Gradient in Polycrystalline Solid Electrolytes
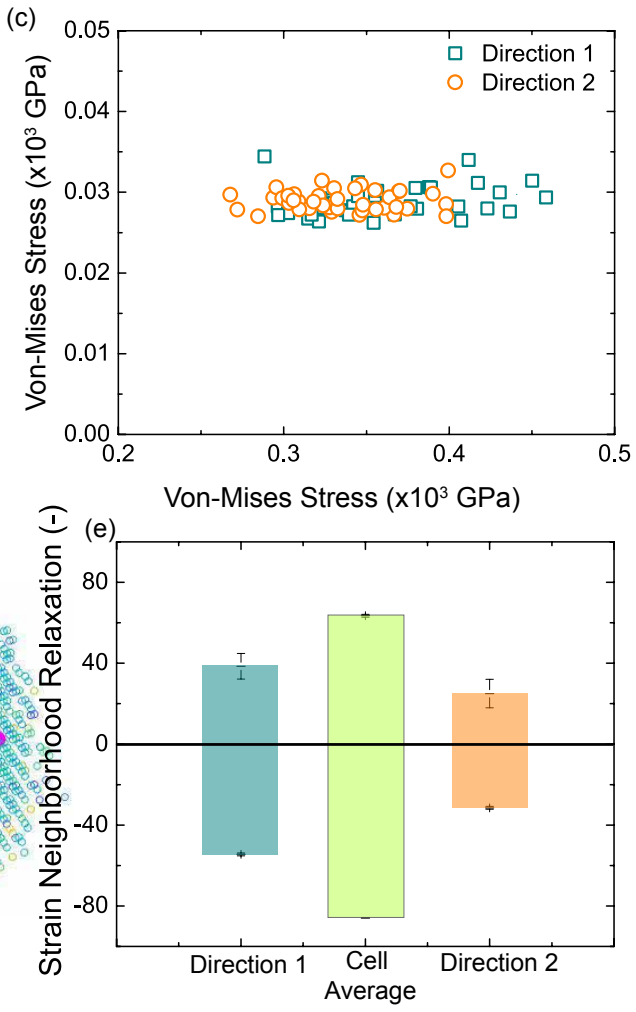

Figure 7: Evaluating Stress Flow Directions in Bulk Solid Electrolytes. (a) Schematic diagram showing the two ion current directions based on the applied electric field. Correlation between (a) Hydrostatic and (b) von-mises stress of the grains separated as function of the field direction. (d) Stress flow pathways identified by the path of maximum gradient for stress within the sample. (e) Average relaxation as a cell average and for the individual field directions. The relaxation is defined as the difference in the Z-position of the initial and the final grain in the stress flow paths.

for individual grains enables to assess the correlation of ion flow and stress flow within the bulk solid electrolyte. For this discussion, two directions are considered within the symmetric cell wherein electrodeposition at the working electrode is assumed as direction 1 of ion flow, while electrodissolution from the working electrode is assumed as direction 2 (Fig. 7a).

Hydrostatic and von-mises are identified and averaged over the hot-/cold-spot neighborhoods from the FF-HEDM dataset for each electrochemical step. Subsequently, these values are plotted so that for each point in the graph, the initial state configuration (pristine, plating-1, stripping-1, plating-2) is the $\mathrm{X}$ co-ordinate and the eventual state configuration (plating-1, stripping-1, plating-2, stripping-2) is the Y co-ordinate. This allows to evaluate if the stress evolution has any influence as dictated by the ion transport direction. The results show that only the hydrostatic stress shows clear separation between the two ion transport direction, while no directional separation was observed for the Von-Mises stress. The hydrostatic stress represents normal stress acting on the grains which is consistent with overall ion transport directions and the clear separation of the normal stress components based on the direction of the ion transport is indicative of interplay of mechanics and ion transport within the solid electrolyte. Specifically, it is proposed that directional stress-induced currents contribute to differences in mechanical response during cycling and thus lead to the separation of the 
distribution observed. To further elucidate this, stress flow pathways from the identified hot-spots are plotted through the sample (Fig. 7d). This is carried out by identifying the nearest grain to the grain of interest, with the highest stress gradient and following it to the minimum value within the bulk. The normalized change in Z-position of the initial and final grain is defined as the stress neighborhood relaxation (Fig. 7e). A positive value of this metric suggests that stress flow is along the direction of counter electrode to working electrode, while a negative value indicates the opposite direction. This analysis is carried out over 50 grains with the highest "hydrostatic" stress value within the solid electrolyte over all the steps of electrochemical cycling. The results show that on average, over multiple cycles and grains in the system, the stress-flow direction favors transport in one direction compared to the other and the separation is stronger for when the field current is along Direction 1, compared to the Direction 2. The results discussed here on differences in distribution of stress flow pathways and stress evolution based on the direction of field-driven transport assert the hypothesis of strong coupling between mechanics and charge-transport within this system.

\section{Conclusion}

This work reports coupled far-field high energy diffraction microscopy and tomography measurements to assess the grain-level chemo-mechanics of garnet solid electrolytes and understanding the origin of failure mechanisms in these materials. Mechanical state mapping of all the grains in bulk solid electrolytes during electrochemical cycling indicates that failure in garnet solid electrolytes is initiated locally, and is likely a stochastic process. Combining FF-HEDM results, high energy X-ray diffraction as well as scattering tomography measurements, the presence of a trace, secondary cubic polymorphic phase of LLZO is identified within the bulk solid electrolytes. This secondary phase is proposed to be the origin of the local, isolated failure mechanisms in garnet solid electrolytes. This hypothesis is supported by measurements of microstructure evolution of stress hot- and cold- spots with the coupled FF-HEDM and tomography dataset as well as finite element modelling. A strong coupling between field-driven ion transport and mechanical response of the grains within the bulk solid electrolytes is also observed. High degree of control of elemental composition during processing and sintering is anticipated as necessary to mitigate the isolated failure mechanisms. Additionally, the result of coupling of stress-transport within solid electrolytes indicate that tailoring residual stress conditions in the bulk solid electrolyte can provide a viable pathway for regularizing electrodeposition of Li metal and engineering of high performing polycrystalline solid electrolytes.

\section{Acknowledgement}

The authors were supported by the National Science Foundation under grant No. 1727863 and 1847029. The authors acknowledge the Vanderbilt Institute of Nanoscience and Engineering (VINSE) for access to their shared characterization facilities. P.P.M. acknowledges support in part from the National Science Foundation (Award No. 2041499). This research 
used resources of the Advanced Photon Source, a U.S. Department of Energy (DOE) Office of Science User Facility operated for the DOE Office of Science by Argonne National Laboratory under Contract No. DE-AC02-06CH11357. This research was carried out in part at Oak Ridge National Laboratory, managed by UT Battelle, LLC, for the U.S. Department of Energy (DOE) under contract DE-AC05-00OR22725. M.B.D. was also supported in part by Alvin M. Weinberg Fellowship at the Oak Ridge National Laboratory.

\section{Author Contributions}

M.B.D. and K.B.H conceived the concept and idea. M.B.D. and W.Z. performed synchrotron imaging and diffraction experiments. J-S.P, J.A, P.K. performed synchrotron measurements and helped with analysis. M.B.D. completed image processing and analysis from synchrotron experiments. B.V., P.M. carried out the modelling efforts. M.D. and K.B.H. wrote the manuscript. The manuscript was edited by all authors.

\section{Declaration of Interests}

There are no conflicts of interest to declare.

\section{References}

[1] Thorben Krauskopf, Felix H. Richter, Wolfgang G. Zeier, and Jürgen Janek. (2020). Physicochemical Concepts of the Lithium Metal Anode in Solid-State Batteries. Chemical Reviews.

[2] Jürgen Janek and Wolfgang G. Zeier. (2016). A solid future for battery development. Nature Energy. 1:16141.

[3] Simon Randau, Dominik A. Weber, Olaf Kötz, Raimund Koerver, Philipp Braun, André Weber, Ellen Ivers-Tiffée, Torben Adermann, Jörn Kulisch, Wolfgang G Zeier, Felix H Richter, and Jürgen Janek. (2020). Benchmarking the performance of all-solid-state lithium batteries. Nature Energy. 5(3):259-270.

[4] Marm B Dixit, Nikhilendra Singh, James P Horwath, Pavel D Shevchenko, Michael Jones, Eric A Stach, Timothy S Arthur, and Kelsey B Hatzell. (2020). jem¿In Situi/emi Investigation of Chemomechanical Effects in Thiophosphate Solid Electrolytes. Matter. 3(6):2138-2159.

[5] Kelsey B. Hatzell, Xi Chelsea Chen, Corie Cobb, Neil P Dasgupta, Marm B. Dixit, Lauren E Marbella, Matthew T. McDowell, Partha Mukherjee, Ankit Verma, Venkatasubramanian Viswanathan, Andrew Westover, and Wolfgang G. Zeier. (2020). Challenges in lithium metal anodes for solid state batteries. ACS Energy Letters. 5:922-934. 
[6] Aashutosh N Mistry and Partha P Mukherjee. (2020). Molar Volume Mismatch : a Malefactor for Irregular Metallic Electrodeposition in Solid Electrolytes. Journal of The Electrochemical Society2. 167(8):082510.

[7] Marm B. Dixit, Jun-Sang Park, Peter Kenesei, Jonathan Almer, and Kelsey B. Hatzell. (2021). Status and prospect of in situ and operando characterization of solid-state batteries. Energy \& Environmental Science. page 10.1039/D1EE00638J.

[8] Marm B. Dixit, Anand Parejiya, Nitin Muralidharan, Rachid Essehli, Ruhul Amin, and Ilias Belharouak. (2021). Understanding implications of cathode architecture on energy density of solid-state batteries. Energy Storage Materials. 40:239-249.

[9] John A. Lewis, Francisco Javier Quintero Cortes, Yuhgene Liu, John C. Miers, Ankit Verma, Bairav S. Vishnugopi, Jared Tippens, Dhruv Prakash, Thomas S. Marchese, Sang Yun Han, Chanhee Lee, Pralav P. Shetty, Hyun-Wook Lee, Pavel Shevchenko, Francesco De Carlo, Christopher Saldana, Partha P. Mukherjee, and Matthew T. McDowell. (2021). Linking void and interphase evolution to electrochemistry in solid-state batteries using operando X-ray tomography. Nature Materials 2021 20:4. 20(4):503-510.

[10] Kelsey B Hatzell and Yanjie Zheng. (2021). Prospects on large-scale manufacturing of solid state batteries. MRS Energy Sustain. 8(1):33-39.

[11] Bairav S. Vishnugopi, Eric Kazyak, John A. Lewis, Jagjit Nanda, Matthew T. McDowell, Neil P. Dasgupta, and Partha P. Mukherjee. (2021). Challenges and Opportunities for Fast Charging of Solid-State Lithium Metal Batteries. ACS Energy Lett. 6(10):37343749 .

[12] Fudong Han, Andrew S Westover, Jie Yue, Xiulin Fan, Fei Wang, Miaofang Chi, Donovan N Leonard, Nancy J Dudney, Howard Wang, and Chunsheng Wang. (2019). High electronic conductivity as the origin of lithium dendrite formation within solid electrolytes. Nature Energy. 4(3):187-196.

[13] Kris Shen. (2018). Enhancing stability between LLZO electrolyte and Li metal electrode by a MXene coating MXene coating on garnet electrolyte.

[14] J Kasemchainan, S Zekoll, D Spencer Jolly, Z Ning, G O Hartley, T J Marrow, and P G Bruce. (2019). Critical stripping current leads to dendrite formation on plating in 3 lithium anode solid electrolyte cells. Nature Materials.

[15] Charles Monroe and John Newman. (2003). Dendrite Growth in Lithium/Polymer Systems A Propagation Model for Liquid Electrolytes Under Galvanostatic Conditions. Journal of The Electrochemical Society. 150(10):A1377-A1384.

[16] Charles Monroe and John Newman. (2005). The Impact of Elastic Deformation on Deposition Kinetics at Lithium/Polymer Interfaces. Journal of The Electrochemical Society. 152(2):A396. 
[17] Michael J. Wang, Rishav Choudhury, and Jeff Sakamoto. (2019). Characterizing the Li-Solid-Electrolyte Interface Dynamics as a Function of Stack Pressure and Current Density. Joule. 3(9):2165-2178.

[18] Nikhilendra Singh, James P Horwath, Patrick Bonnick, Koji Suto, Eric A. Stach, Tomoya Matsunaga, John Muldoon, and Timothy S. Arthur. (2020). The role of lithium iodide addition to lithium thiophosphate: Implications beyond conductivity. Chemistry of Materials.

[19] Tetsuya Asano, Akihiro Sakai, Satoru Ouchi, Masashi Sakaida, Akinobu Miyazaki, and Shinya Hasegawa. (2018). Solid Halide Electrolytes with High Lithium-Ion Conductivity for Application in $4 \mathrm{~V}$ Class Bulk-Type All-Solid-State Batteries. Advanced Materials. $30(44)$.

[20] Jin Zheng and Yan-yan Hu. (2018). New Insights into the Compositional Dependence of Li-Ion Transport in Polymer Ceramic Composite Electrolytes. ACS Applied Materials E Interfaces. 10(4):4113-4120.

[21] Jitti Kasemchainan and G Peter. (2018). All-Solid-State Batteries and their Remaining Challenges. Johnson Matthey Technology Review. 2(2):177-180.

[22] Lukas Porz, Tushar Swamy, Brian W Sheldon, Daniel Rettenwander, Till Frömling, Henry L Thaman, Stefan Berendts, Reinhard Uecker, W Craig Carter, and Yet-Ming Chiang. (2017). Mechanism of Lithium Metal Penetration Through Inorganic Solid Electrolytes. Advanced Energy Materials. 7(20):1701003.

[23] Sebastian Wenzel, Stefan J. Sedlmaier, Christian Dietrich, Wolfgang G. Zeier, and Juergen Janek. (2018). Interfacial reactivity and interphase growth of argyrodite solid electrolytes at lithium metal electrodes. Solid State Ionics. 318(February 2017):102-112.

[24] Raimund Koerver, Isabel Aygu, Thomas Leichtweiß, Christian Dietrich, Wenbo Zhang, Jan O Binder, Pascal Hartmann, Wolfgang G Zeier, and Ju Janek. (2017). Capacity Fade in Solid-State Batteries : Interphase Formation and Chemomechanical Processes in Nickel-Rich Layered Oxide Cathodes and Lithium Thiophosphate Solid Electrolytes.

[25] John A Lewis, Francisco Javier, Quintero Cortes, Matthew G Boebinger, Jared Tippens, Thomas S Marchese, Neha Kondekar, Xiaoming Liu, Miaofang Chi, and Matthew T Mcdowell. (2019). Interphase Morphology between a Solid-State Electrolyte and Lithium Controls Cell Failure. ACS Energy Letters. 4:591-599.

[26] Ziyang Ning, Dominic Spencer Jolly, Guanchen Li, Robin De Meyere, Shengda D. Pu, Yang Chen, Jitti Kasemchainan, Johannes Ihli, Chen Gong, Boyang Liu, Dominic L. R. Melvin, Anne Bonnin, Oxana Magdysyuk, Paul Adamson, Gareth O. Hartley, Charles W. Monroe, T. James Marrow, and Peter G. Bruce. (2021). Visualizing platinginduced cracking in lithium-anode solid-electrolyte cells. Nature Materials 2021 20:8. 20(8):1121-1129. 
[27] Jared Tippens, John C. Miers, Arman Afshar, John A. Lewis, Francisco Javier Quintero Cortes, Haipeng Qiao, Thomas S. Marchese, Claudio V. Di Leo, Christopher Saldana, and Matthew T. McDowell. (2019). Visualizing Chemomechanical Degradation of a Solid-State Battery Electrolyte. ACS Energy Letters. 4(6):1475-1483.

[28] J Kasemchainan, S Zekoll, D Spencer Jolly, Z Ning, G O Hartley, T J Marrow, and P G Bruce. (2019). Critical stripping current leads to dendrite formation on plating in 3 lithium anode solid electrolyte cells. Nature Materials.

[29] Dominic Spencer Jolly, Ziyang Ning, James E. Darnbrough, Jitti Kasemchainan, Gareth O. Hartley, Paul Adamson, David E.J. Armstrong, James Marrow, and Peter G. Bruce. (2020). Sodium/Na $\beta$ Alumina Interface: Effect of Pressure on Voids. ACS Applied Materials and Interfaces. 12(1):678-685.

[30] Yutao Li, Xi Chen, Andrei Dolocan, Zhiming Cui, Sen Xin, Leigang Xue, Henghui Xu, Kyusung Park, and John B. Goodenough. (2018). Garnet Electrolyte with an Ultralow Interfacial Resistance for Li-Metal Batteries. Journal of the American Chemical Society. 140(20):6448-6455.

[31] Jiajia Tan and Ashutosh Tiwari. (2012). Synthesis of Cubic Phase Li 7 La 3 Zr 2 O 12 Electrolyte for Solid-State Lithium-Ion Batteries. Electrochemical and Solid-State Letters. 15(3):37-39.

[32] Bing Sun, Chao Xu, Jonas Mindemark, Torbjörn Gustafsson, Kristina Edström, and Daniel Brandell. (2015). At the polymer electrolyte interfaces: the role of the polymer host in interphase layer formation in Li-batteries. J. Mater. Chem. A. 3(26):1399414000 .

[33] Hany El-Shinawi, Gary W. Paterson, Donald A. MacLaren, Edmund J. Cussen, and Serena A. Corr. (2017). Low-temperature densification of Al-doped Li isubi $7 i /$ subi La isubi3i/subi, Zr isubi2i/subi, O isubi12i/subi : a reliable and controllable synthesis of fast-ion conducting garnets. J. Mater. Chem. A. 5(1):319-329.

[34] Daniel Rettenwander, Gu Redhammer, Florian Preishuber-p, Lei Cheng, Lincoln Miara, Reinhard Wagner, Andreas Welzl, Emmanuelle Suard, Marca M Doe, Martin Wilkening, Ju Fleig, and Georg Amthauer. (2016). Structural and Electrochemical Consequences of $\mathrm{Al}$ and Ga Cosubstitution in Li 7 La 3 Zr 2 O 12 Solid Electrolytes.

[35] Jay C. Schuren, Paul A. Shade, Joel V. Bernier, Shiu Fai Li, Basil Blank, Jonathan Lind, Peter Kenesei, Ulrich Lienert, Robert M. Suter, Todd J. Turner, Dennis M. Dimiduk, and Jonathan Almer. (2015). New opportunities for quantitative tracking of polycrystal responses in three dimensions. Current Opinion in Solid State and Materials Science. 19(4):235-244.

[36] Seungho Yu, Robert D Schmidt, Regina Garcia-Mendez, Erik Herbert, Nancy J Dudney, Jeffrey B Wolfenstine, Jeff Sakamoto, and Donald J Siegel. (2015). Elastic Properties of the Solid Electrolyte Li7La3Zr2O12 (LLZO). Chemistry of Materials. 28(1):197-206. 
[37] Chuang Yu, Swapna Ganapathy, Ernst R.H.Van Eck, Heng Wang, Shibabrata Basak, Zhaolong Li, and Marnix Wagemaker. (2017). Accessing the bottleneck in all-solid state batteries, lithium-ion transport over the solid-electrolyte-electrode interface. Nature Communications. 8(1):1-9.

[38] Asma Sharafi, Harry M. Meyer, Jagjit Nanda, Jeff Wolfenstine, and Jeff Sakamoto. (2016). Characterizing the Li-Li7La3Zr2O12interface stability and kinetics as a function of temperature and current density. Journal of Power Sources. 302:135-139.

[39] Asma Sharafi, Catherine G Haslam, Robert D Kerns, Jeff Wolfenstine, and Jeff Sakamoto. (2017). Controlling and Correlating the Effect of Grain Size with the Mechanical and Electrochemical Properties of Li7La3Zr2O12 Solid-State Electrolyte. Journal of Materials Chemistry A. 5(40):21491-21504.

[40] L Cheng, W Chen, M Kunz, K Persson, N Tamura, G Chen, and M Doeff. (2015). Effect of Surface Microstructure on Electrochemical Performance of Garnet Solid Electrolytes. ACS Applied Materials \& Interfaces. 7(3):2073-2081.

[41] Xin Xu, Yuzi Liu, Jie Wang, Dieter Isheim, Vinayak P. Dravid, Charudatta Phatak, and Sossina M. Haile. (2020). Variability and origins of grain boundary electric potential detected by electron holography and atom-probe tomography. Nature Materials. 19:887893.

[42] Marm Dixit, Nikhilendra Singh, James Horwath, Pavel Shevchenko, Eric Stach, Timothy S Arthur, and Kelsey Hatzell. (2020). In Situ Investigation of Interphase and Microstructure Effects on the Chemo-Mechanics of Thiophosphate Solid Electrolytes.

[43] Marm B Dixit, Ankit Verma, Wahid Zaman, Xinlin Zhong, Peter Kenesei, Jun Sang Park, Jonathan Almer, Partha P Mukherjee, and Kelsey B Hatzell. (2020). Synchrotron Imaging of Pore Formation in Li Metal Solid-State Batteries Aided by Machine Learning. ACS Applied Energy Materials. 3(10):9534-9542.

[44] Ying Zhang, Yang Shi, Xin Cheng Hu, Wen Peng Wang, Rui Wen, Sen Xin, and Yu Guo Guo. (2020). A 3D Lithium/Carbon Fiber Anode with Sustained Electrolyte Contact for Solid-State Batteries. Advanced Energy Materials. 10(3):1-8.

[45] Sooraj Narayan and Lallit Anand. (2020). On Modeling the Detrimental Effects of Inhomogeneous Plating-and-Stripping at a Lithium-Metal/Solid-Electrolyte Interface in a Solid-State-Battery. Journal of The Electrochemical Society. 167(4):040525.

[46] Michael Wang and Jeff Sakamoto. (2018). Correlating the interface resistance and surface adhesion of the Li metal-solid electrolyte interface. Journal of Power Sources. 377(November 2017):7-11.

[47] Jun-Sang Park, Xuan Zhang, Peter Kenesei, Su Leen Wong, Meimei Li, and Jonathan Almer. (2017). Far-Field High-Energy Diffraction Microscopy: A Non-Destructive Tool for Characterizing the Microstructure and Micromechanical State of Polycrystalline Materials. Microscopy Today. 25(5):36-45. 
[48] Mie Elholm Birkbak, Ida Gjerlevsen Nielsen, Simon Frølich, Stuart R Stock, Peter Kenesei, Jonathan D Almer, and Henrik Birkedal. (2017). Concurrent Determination of Nanocrystal Shape and Amorphous Phases in Complex Materials by Diffraction Scattering Computed Tomography. 50.

[49] Marm Dixit. (2018). Analytical modeling of coating windows. pages $1-15$.

[50] Marm B. Dixit, Daniel Moreno, Xianghui Xiao, Marta C. Hatzell, and Kelsey B. Hatzell. (2019). Mapping Charge Percolation in Flowable Electrodes Used in Capacitive Deionization. ACS Materials Letters. page acsmaterialslett.9b00106.

[51] Marm B. Dixit, Wahid Zaman, Nicholas Hortance, Stella Vujic, Brice Harkey, Fengyu Shen, Wan Yu Tsai, Vincent De Andrade, X. Chelsea Chen, Nina Balke, and Kelsey B. Hatzell. (2020). Nanoscale Mapping of Extrinsic Interfaces in Hybrid Solid Electrolytes. Joule. 4(1):207-221.

[52] R. V. Martins, C. Ohms, and K. Decroos. (2010). Full 3D spatially resolved mapping of residual strain in a 316L austenitic stainless steel weld specimen. Materials Science and Engineering A. 527(18-19):4779-4787.

[53] J. S. Park, U. Lienert, P. R. Dawson, and M. P. Miller. (2013). Quantifying Three-Dimensional Residual Stress Distributions Using Spatially-Resolved Diffraction Measurements and Finite Element Based Data Reduction. Experimental Mechanics. 53(9):1491-1507.

[54] D. R. Haeffner, J. D. Almer, and U. Lienert. (2005). The use of high energy X-rays from the Advanced Photon Source to study stresses in materials. Materials Science and Engineering A. 399(1-2):120-127.

[55] S. F. Nielsen, A. Wolf, H. F. Poulsen, M. Ohler, U. Lienert, and R. A. Owen. (2000). A conical slit for three-dimensional XRD mapping. Journal of Synchrotron Radiation. $7(2): 103-109$.

[56] R. M. Suter, D. Hennessy, C. Xiao, and U. Lienert. (2006). Forward modeling method for microstructure reconstruction using x-ray diffraction microscopy: Single-crystal verification. Review of Scientific Instruments. 77(12):123905.

[57] U. Lienert, J. Lind, C. M. Hefferan, W. Pantleon, M. J. Mills, M. P. Miller, R. M. Suter, S. F. Li, M. C. Brandes, J. V. Bernier, B. Jakobsen, and N. R. Barton. (2011). High-energy diffraction microscopy at the Advanced Photon Source. JOM. 63(7):70-77.

[58] S. Schmidt and IUCr. (2014). GrainSpotter: a fast and robust polycrystalline indexing algorithm. urn:issn:1600-5767. 47(1):276-284.

[59] J V Bernier, N R Barton, U Lienert, and M P Miller. (2011). Far-field highenergy diffraction microscopy: a tool for intergranular orientation and strain analysis:. http://dx.doi.org/10.1177/0309324711405761. 46(7):527-547. 
[60] Justin M. Wozniak, Hemant Sharma, Timothy G. Armstrong, Michael Wilde, Jonathan D. Almer, and Ian Foster. (2015). Big Data Staging with MPI-IO for Interactive X-ray Science. Proceedings - 2014 International Symposium on Big Data Computing, BDC 2014. pages 26-34.

[61] Hemant Sharma, Richard M. Huizenga, and S. Erik Offerman. (2012). A fast methodology to determine the characteristics of thousands of grains using three-dimensional $\mathrm{X}$-ray diffraction. II. Volume, centre-of-mass position, crystallographic orientation and strain state of grains. Journal of Applied Crystallography. 45(4):705-718.

[62] Hemant Sharma, Richard M. Huizenga, and S. Erik Offerman. (2012). A fast methodology to determine the characteristics of thousands of grains using three-dimensional $\mathrm{X}$-ray diffraction. I. Overlapping diffraction peaks and parameters of the experimental setup. Journal of Applied Crystallography. 45(4):693-704.

[63] H F Poulsen, (2004). Three-Dimensional X-Ray Diffraction Microscopy: Mapping Polycrystals and Their Dynamics. Springer Tracts in Modern Physics. Springer.

[64] S Yu, R D Schmidt, R Garcia-Mendez, E Herbert, N J Dudney, J B Wolfenstine, J Sakamoto, and D J Siegel. (2016). Elastic Properties of the Solid Electrolyte Li7La3Zr2O12 (LLZO). Chemistry of Materials. 28(1):197-206.

[65] Bairav Sabarish Vishnugopi, Feng Hao, Ankit Verma, and Partha P. Mukherjee. (2020). Double-Edged Effect of Temperature on Lithium Dendrites. ACS Appl. Mater. Interfaces. 12(21):23931-23938.

[66] Bairav S. Vishnugopi, Feng Hao, Ankit Verma, and Partha P. Mukherjee. (2020). Surface diffusion manifestation in electrodeposition of metal anodes. Phys. Chem. Chem. Phys. 22(20):11286-11295.

[67] Carlos Bernuy-Lopez, William Manalastas, Juan Miguel Lopez del Amo, Ainara Aguadero, Frederic Aguesse, and John A Kilner. (2014). Atmosphere Controlled Processing of Ga-Substituted Garnets for High Li-Ion Conductivity Ceramics. Chem. Mater. 26(12):3610-3617.

[68] Xiao Huang, Yang Lu, Zhen Song, Tongping Xiu, Michael E. Badding, and Zhaoyin Wen. (2019). Preparation of dense Ta-LLZO/MgO composite Li-ion solid electrolyte: Sintering, microstructure, performance and the role of MgO. Journal of Energy Chemistry. 39:8-16.

[69] Jianmeng Su, Xiao Huang, Zhen Song, Tongping Xiu, Michael E. Badding, Jun Jin, and Zhaoyin Wen. (2019). Overcoming the abnormal grain growth in Ga-doped Li7La3Zr2O12 to enhance the electrochemical stability against Li metal. Ceramics International. 45(12):14991-14996.

[70] Theodosios Famprikis, Pieremanuele Canepa, James A. Dawson, M. Saiful Islam, and Christian Masquelier. (2019). Fundamentals of inorganic solid-state electrolytes for batteries. Nature Materials. 18(12):1278-1291. 
[71] Reinhard Wagner, Günther J. Redhammer, Daniel Rettenwander, Anatoliy Senyshyn, Walter Schmidt, Martin Wilkening, and Georg Amthauer. (2016). Crystal Structure of Garnet-Related Li-Ion Conductor Li7-3xGaxLa3Zr2O12: Fast Li-Ion Conduction Caused by a Different Cubic Modification? Chem. Mater. 28(6):1861-1871.

[72] Dominic Spencer Jolly, Ziyang Ning, Gareth O. Hartley, Boyang Liu, Dominic L. R. Melvin, Paul Adamson, James Marrow, and Peter G. Bruce. (2021). Temperature Dependence of Lithium Anode Voiding in Argyrodite Solid-State Batteries. ACS Applied Materials Interfaces. 13(19):22708-22716. 


\title{
Supporting Information: Polymorphism of Garnet Solid Electrolytes and Its Implications on Grain Level Chemo-Mechanics
}

\author{
Marm B. Dixit * ${ }^{* \ddagger} \quad$ Bairav Vishugopi ${ }^{\S} \quad$ Wahid Zaman ${ }^{\dagger}$ \\ Peter Keneseill Jun-Sang Park ${ }^{\ddagger} \quad$ Jonathan Almer \\ Partha Mukherjee ${ }^{\dagger} \quad$ Kelsey B. Hatzell ${ }^{* \dagger * * *}$
}

This manuscript has been authored by UT-Battelle, LLC, under Contract No. DEAC0500OR22725 with the U.S. Department of Energy. The United States Government retains and the publisher, by accepting the article for publication, acknowledges that the United States Government retains a non-exclusive, paid-up, irrevocable, world-wide license to publish or reproduce the published form of this manuscript, or allow others to do so, for the United States Government purposes. The Department of Energy will provide public access to these results of federally sponsored research in accordance with the DOE Public Access Plan (http://energy.gov/downloads/doe-public-access-plan).

${ }^{*}$ Lead Contact and Corresponding Author: kelsey.hatzell@princeton.edu and dixitmb@ornl.gov

${ }^{\dagger}$ Department of Mechanical Engineering, Vanderbilt University, Nashville, TN, 37240, USA

${ }^{\ddagger}$ Electrification and Energy Infrastructures Division, Oak Ridge National Laboratory, Oak Ridge, TN, 37830, USA

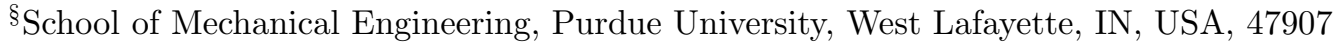

ILead Contact and Corresponding Author: kelsey.hatzell@princeton.edu and dixitmb@ornl.gov

"X-ray Science Division, Argonne National Laboratory, 9700 South Cass Avenue, Lemont, Illinois 60439, USA

**Andlinger Center for Energy and Environment, Princeton University, Princeton, NJ, 08540, USA 


\section{Supplemental Experimental Procedures}

\section{S1. Electrolyte, Cell Preparation and Electrochemical Testing}

$\mathrm{Li}_{6.5} \mathrm{La}_{3} \mathrm{Zr}_{1.5} \mathrm{Ta}_{0.5} \mathrm{O}_{12}$ was synthesized using solid state reaction process as detailed pre-

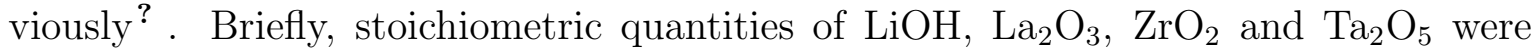
ball-milled and sintered initially. Subsequent to a secondary ball-milling cycle for sizereduction, $3 \mathrm{~mm}$ diameter pellets were pressed and sintered at $1130{ }^{\circ} \mathrm{C}$ for 10 hours. The pellets were subsequently polished to a diameter of approximately $1.6 \mathrm{~mm}$ for synchrotron experiments. The maximum sample size is determined by the optics configuration, sample-detector distance, d-spacing range under investigation as well as the beam energy. The $\approx 2 \mathrm{~mm}$ size of the sample was optimized to provide high resolution for strain over the d-spacing range of interest in LLZO as well as enable Li metal imaging. In our earlier work, we have discussed the role of the experimental constraints and its impact on the cause for the relatively low values of the critical current density metrics measured in in situ conditions. Symmetric Li|LLZO|Li cells were assembled in an argon glove box at the Advanced Photon Source. The cell was loaded onto a special in situ cell to enable electrochemical testing while carrying out diffraction and tomography experiments. Galvanostatic plating/stripping tests were carried out on $\mathrm{Li}|\mathrm{LLZO}| \mathrm{Li}$ symmetric cells at increasing current density until the short-circuit of the cell was observed. Electronic impedance spectroscopy was carried out between $1 \mathrm{MHz}$ and $100 \mathrm{mHz}$ with a $50 \mathrm{mV}$ amplitude. Impedance measurements were taken after individual plating and stripping cycles.

\section{S2. HEDM and Tomography Measurements}

Far Field high energy diffraction microscopy (FF-HEDM) experiments were carried out at 1-ID-E beamline of the Advanced Photon Source at the Argonne National Laboratory (Fig. S1). Experimental conditions for LLZTO were identified with 76.2 $\mathrm{keV}$ and $2.189 \mathrm{~m}$ as the optimum energy and sample-detector distance. In a typical FF-HEDM experiment, the sample was rotated from -180 to 180 degree with respect to incoming X-rays at $0.1^{\circ}$ steps. Diffraction images were captured on a $2 \mathrm{D}$ area detector (GE-41RT, 2048x2048 pixels, $200 \mu \mathrm{m}$ pitch) with $1 \mathrm{~s}$ exposure time. FF-HEDM used a box beam of $100 \mu \mathrm{m}$ height and hence the scan for the entire Li|LLZO|Li cell was completed in over 9 sections. Acquisition time for a single FF-HEDM scan of the entire LLZO cell was roughly 1.5 hours. Initially, pristine LLZO cell was characterized with FF-HEDM to assess the initial stress configuration. Subsequently, in-situ testing was carried out for symmetric cells at the end of each plating and stripping step. Tomography on the symmetric cell was also carried out on the cell at interim locations where FF-HEDM scans were taken. Details of the tomography experiment are reported previously ${ }^{1}$.

\section{S3. Synchrotron XRD Measurement}


High resolution XRD patterns were collected from LLZO pellet at 1-ID-E beamline of the Advanced Photon Source at the Argonne National Laboratory. Spatially resolved XRD patterns were collected with a resolution of $0.1 \mathrm{~mm}$. Diffraction was recorded during $180^{\circ}$ rotation of the sample with an exposure time of $0.2 \mathrm{~s}$. The diffraction patterns were recorded on Pilatus CdTe detector which has a dynamic range of 2E20 and a maximum radial dispersity of 100:1. The high dynamic range of the Pilatus allows for capturing diffraction peaks with very low structure factors.

\section{S4. Data Analysis}

Grains were indexed from the FF-HEDM data using the fourth through seventh DebyeScherrer rings corresponding to the $\{231\},\{040\},\{240\}$ and $\{332\}$ lattice planes. The inner rings were avoided for indexing as they were attenuated by filters to reduce saturation of the detector. This also enables to acquire higher intensity on the low d-spacing outer rings to enable high strain resolution. Calibration, reconstruction and indexing was carrying out using the Microstructural Imaging and Diffraction Analysis Software (MIDAS). Reconstruction of the FF-HEDM data yielded the grain centroid position, grain averaged orientation and an elastic strain tensor. Grain identification is carried out by a procedure called indexing which is described here (Fig. S2). Starting with a diffraction spot, its position with respect to the ideal diffraction ring for grains positioned at $(0,0,0)$ lab is calculated. A number of different combinations of centreof-mass position and orientation of the diffracting grain can give rise to this observed position of the diffraction spot. This results in a number of possible positions of the diffracting grain, each with a different orientation of the diffracting plane normal and each of which can give rise to the observed diffraction spot but which, if placed at $(0,0$, 0)lab, would result in a diffraction spot at one of the points on the ideal diffraction ring. Now, considering one of these points, the diffracting grain (with a fixed orientation of the diffracting plane normal) can be located not only at this point but at any position along the diffracted ray passing through this point and the observed diffraction spot. The combination of all these positions in the sample lying on diffracted rays to the observed diffraction spot and passing through the points found on the plane $\mathrm{AA}_{0}$ defines a surface. The surface thus calculated is divided into a grid, and diffraction spots for all combinations of positions on the grid and orientations (by rotating the diffracting plane about its normal) are simulated and matched with the observed diffraction spots, using margins for the position on the detector, the position in and the volume of the grain. The combination of position and orientation with the best match is regarded as belonging to the grain in question. The criteria for estimating closeness of simulated and observed reflection is called completeness, which is a measure of grain tracking through the full sample rotation. Minimum completeness threshold is set at $70 \%$ for the reconstruction.

Typical resolution for centroid positions is approximately $20 \mu \mathrm{m}$, angular position is $0.1^{\circ}$ and lattice strain resolution is $1 \mathrm{E}-4$. Approximately 31000 grains were identified in a single pellet and are consistent across each measurement. The lattice strains are calculated based on the distortion of the crystal lattice from the reference crystal lattice 
spacing.

For each grain, FF-HEDM can simultaneously track the elastic strain as a perturbation of the local lattice. Strain is computed as the difference in lattice parameters of an unstrained lattice to a strained lattice (Fig. ??a, Sx). The direct space metric tensor, is a method of measuring distances in non-cartesian coordinate system. For an typical lattice, the direct space metric tensor is given as,

$$
A=\left(\begin{array}{ccc}
a & b \cos (\gamma) & \cos (\beta) \\
0 & b \sin (\gamma) & -\operatorname{csin}(\beta) \cos \left(\alpha^{*}\right) \\
0 & 0 & c \sin (\beta) \sin \left(\alpha^{*}\right)
\end{array}\right)
$$

Where, $(\mathrm{a}, \mathrm{b}, \mathrm{c}, \alpha, \beta, \gamma)$ and $\left(\mathrm{a}^{*}, \mathrm{~b}^{*}, \mathrm{c}^{*}, \alpha^{*}, \beta^{*}, \gamma^{*}\right)$ are the direct and reciprocal space lattice parameters. The linear Langrangian strain tensor equation defines the strain for the lattice as follows:

$$
\epsilon_{i j}=\frac{1}{2}\left(A A_{0}^{-} 1+A_{0}^{-} 1 A\right)-I
$$

Where $\mathrm{A}$ and $\mathrm{A}_{0}$ are the direct space metric tensors for strained and unstrained lattice respectively while $\mathrm{I}$ is an identity matrix. The lattice parameters are estimated during indexing routines to identify grain locations and orientations. This information is subsequently used with the formulation described here to estimate the elastic strain development in the grains.

For the purposes of this study, the hydrostatic stress is typically reported as a grainaveraged quantity. Grain tracking between individual measurements is carried out by one-to-one correspondence of minimal distances between grain sets. All analysis of grain neighborhoods is carried out on the tracked data-sets enabling a direct spatial correlation between different measurements. In addition to spatial correlation between successive HEDM measurements, grain sets were also registered with corresponding tomography dataset using bounding dimensions as the registration metrics. All data analysis tasks are performed using custom-built routines in MATLAB. Specifically, kmeans clustering $(\mathrm{k}=3)$ was used to identify spatial correlation between strain evolution during electrochemical cycling. Strain relaxation pathways are identified on clusteraveraged data by identifying location of steepest gradient from an identified location. The relaxation pathways are concluded when a local minima is reached. A local minima in this case refers to a grain neighborhood pair that show highest strain gradient when defined from either neighborhood.

\section{S5. Computational Methods}

\section{Kinetic Monte Carlo Model:}

Ion transport behavior at the vicinity of the interface between the bulk and foreign solid electrolyte phases has been captured using a kinetic Monte Carlo modeling approach.

Firstly, transport rate constants, $k_{T 1}$ and $k_{T 2}$ are defined based on ion migration barriers, $E_{a 1}$ and $E_{a 2}$ in the bulk and foreign phases of the solid electrolyte respectively. 
The rate of ion migration from one lattice site to another is calculated according to the Arrhenius equation:

$$
k_{T i}=v \exp \left(\frac{-E_{a i}}{k_{b} T}\right) \quad[i=1,2]
$$

Here, $v$ is the hopping frequency, $T$ is the temperature and $k_{b}$ is the Boltzmann constant.

A total rate constant, $k_{\text {total }}$ is calculated as follows:

$$
k_{\text {total }}=\sum_{i=1}^{N_{1}} k_{T 1}^{i}+\sum_{j=1}^{N_{2}} k_{T 2}^{j}
$$

$N_{1}$ and $N_{2}$ represent the total number ions inside the bulk and foreign phases of the solid electrolyte respectively. Subsequently, a random number $r_{1}$, is chosen between 0 and 1 . All the possible ion migration events in the system are sequentially scanned, and the first event for which the total rate of previously scanned events is larger than $k_{\text {total }} r_{1}$ is selected. The system is then evolved based on the chosen ion transport event. Simulations have been carried out until a total of $4 \times 10^{8}$ ion migration events have been completed. The simulated system is $60 \mathrm{~nm} \times 60 \mathrm{~nm}$, with the foreign phase occupying a $7 \mathrm{~nm} \times 60 \mathrm{~nm}$ region in the middle of the domain. The remainder of the domain is occupied by the bulk solid electrolyte phase. The initial ion occupancy is set to $2 \%$ throughout the domain. The ion migration barriers are taken to be $0.35 \mathrm{eV}$ and $0.30 \mathrm{eV}$ in the bulk and foreign phases respectively.

\section{Mechanics Model:}

To capture the stress state of the system, the governing equation for mechanical stress assuming quasi-static mechanical equilibrium is solved:

$$
\nabla \cdot \boldsymbol{\sigma}=0
$$

Top and bottom boundaries of the solid electrolyte and Li metal respectively are subjected to constant external pressure, $\boldsymbol{\sigma} \boldsymbol{n}=P_{\text {ext }} \boldsymbol{n}$. Left and right boundaries of the solid electrolyte and Li metal are fixed in the normal direction. An external pressure of $1 \mathrm{MPa}$ is applied and linear elasticity is assumed for the lithium metal and solid electrolyte:

$$
\boldsymbol{\sigma}=\frac{E}{1+\nu} \varepsilon+\frac{\nu E}{(1+\nu)(1-2 \nu)} \operatorname{trace}(\varepsilon) \boldsymbol{I}
$$

Here, $\boldsymbol{\sigma}$ and $\varepsilon$ correspond to the stress and strain tensors respectively. The Young's modulus, $E$ of Li is taken to be $7.8 \mathrm{GPa}$, while the Young's modulus of the bulk and foreign phases of LLZO are assumed to be $161 \mathrm{GPa}$ and $156 \mathrm{GPa}$ respectively. The Poisson's ratio $\nu$ of $\mathrm{Li}$ and LLZO are considered as 0.38 and 0.27 respectively. 
Dimensions of the described domain have been represented in the schematic below (Fig. S17); stress state of the dotted region, present away from the interface and consisting of the bulk phase (with the embedded foreign phase) has been plotted in the manuscript. Foreign phases of LLZO, possessing distinct mechanical properties are assumed to be $0.5 \mu \mathrm{m}$ in radius. Co-ordinates of the centers of the circles $c_{1}, c_{2}$ and $c_{3}$ shown in Figure 1 are $(8 \mu \mathrm{m}, 17 \mu \mathrm{m}),(5.5 \mu \mathrm{m}, 12.5 \mu \mathrm{m})$ and $(2 \mu \mathrm{m}, 15.5 \mu \mathrm{m})$ respectively.

\section{Electrochemical-Transport Model:}

The electric potential in the solid electrolyte system is obtained by solving the following governing equation:

$$
\nabla \cdot\left(k_{S E} \nabla \phi_{e}\right)=0
$$

Here, $\phi_{e}$ represents the electric potential and $k_{S E}$ represents the ionic conductivity of the solid electrolyte, taken to be $0.3 \mathrm{mS} / \mathrm{cm}$ and $1 \mathrm{mS} / \mathrm{cm}$ in the bulk and foreign phases respectively.

At the Li-electrolyte interface, electrochemical reactions following the Butler-Volmer kinetics occur: $-k_{S E} \nabla \phi_{e}=i_{0}\left(\exp \left(\frac{\alpha_{a} F}{R T} \eta\right)-\exp \left(-\frac{\alpha_{c} F}{R T} \eta\right)\right)$.Here, $i_{0}$ is the exchange current density, $F$ is the Faraday constant, $R$ is the Gas constant, T is the temperature, $\alpha_{a}$ and $\alpha_{c}$ are the charge transfer coefficients and $\eta$ is the kinetic overpotential. Due to the high electronic conductivity of lithium metal, potential drop in Li metal domain has been neglected in the calculations. Current density $\left(i_{a p p}=0.5 \mathrm{~mA} / \mathrm{cm}^{2}\right)$ is applied at the top boundary of the solid electrolyte, $-k_{S E} \nabla \phi_{e}=i_{a p p}$. The left and right boundaries are set to: $\nabla \phi_{e} \cdot \boldsymbol{n}=0$

The electric potential field at the vicinity of the foreign phase region located at $(5.5 \mu \mathrm{m}$, $12.5 \mu \mathrm{m})$ has been presented in the manuscript. 
(a) APS 1-ID Beamline Specifications:

Monochromatic Beam: 42-120 keV (single energy)

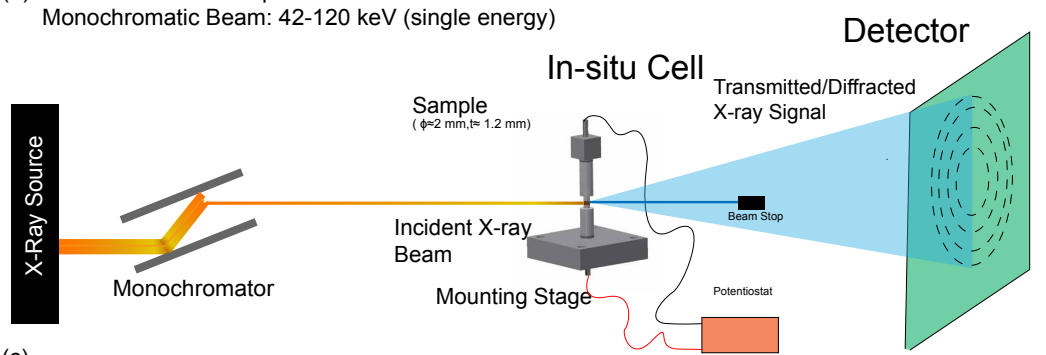

(b)
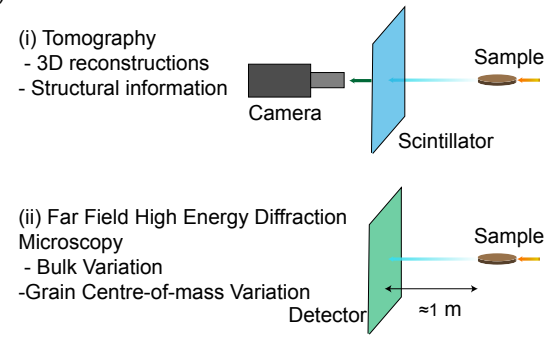

(c)

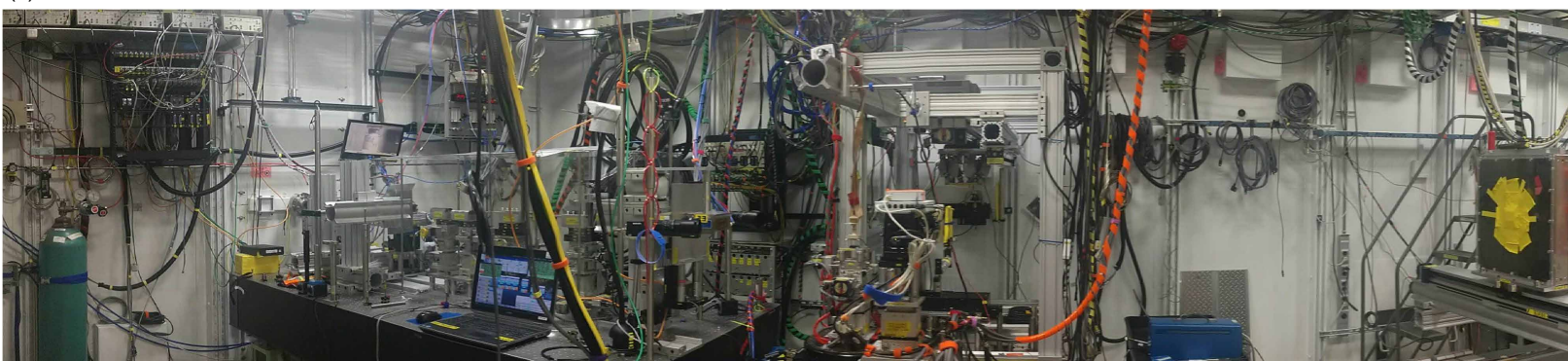

Focusing Optics

Sample Stage

Detector

Fig S 1: Far field and tomography Measurement Experimental Setup. (a) Schematic diagram showing experimental setup with the relevant equipments highlighted. (b) End-station detectors for carrying out tomography and far-field high enregy diffraction microscopy measurements with the corresponding information expected. (c) Photograph of the experimental end-station at the Advanced Photon Source, 1-ID-E end station. The beam enters the hutch and passes through the end-station focusing optics before being incident on the sample. The diffracted signal are captured on a large area detector.

(a)

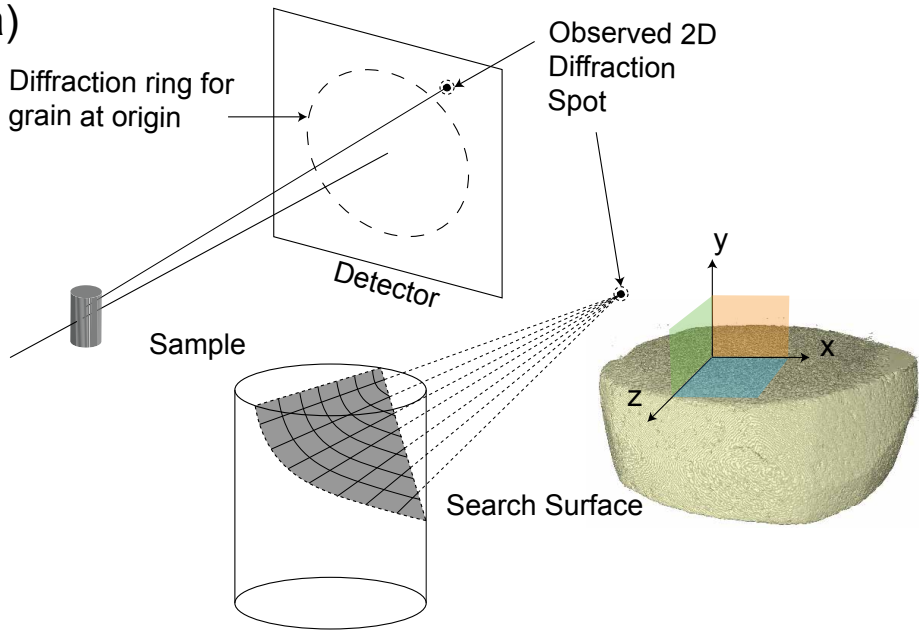

(b)

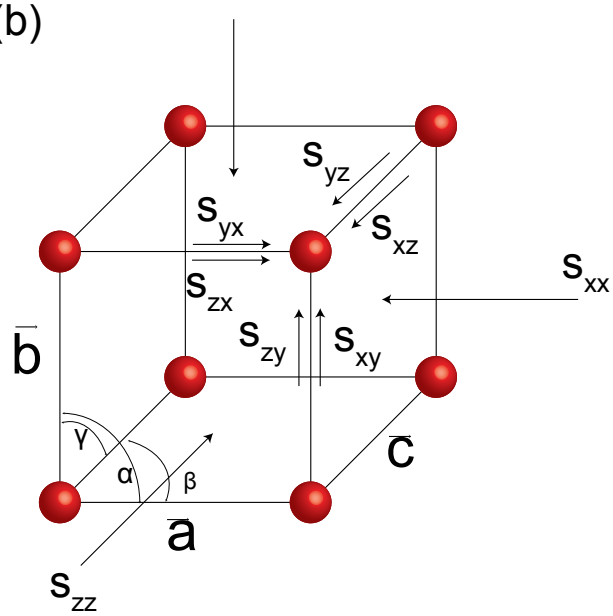

Fig S 2: Indexing process and data reduction for FF-HEDM. (a) Schematic diagram explaining indexing method for identifying grain orientation and COM. (b)Nomenclature for the strain tensor and correlation of the spatial co-ordinates with geometry. 
(a)
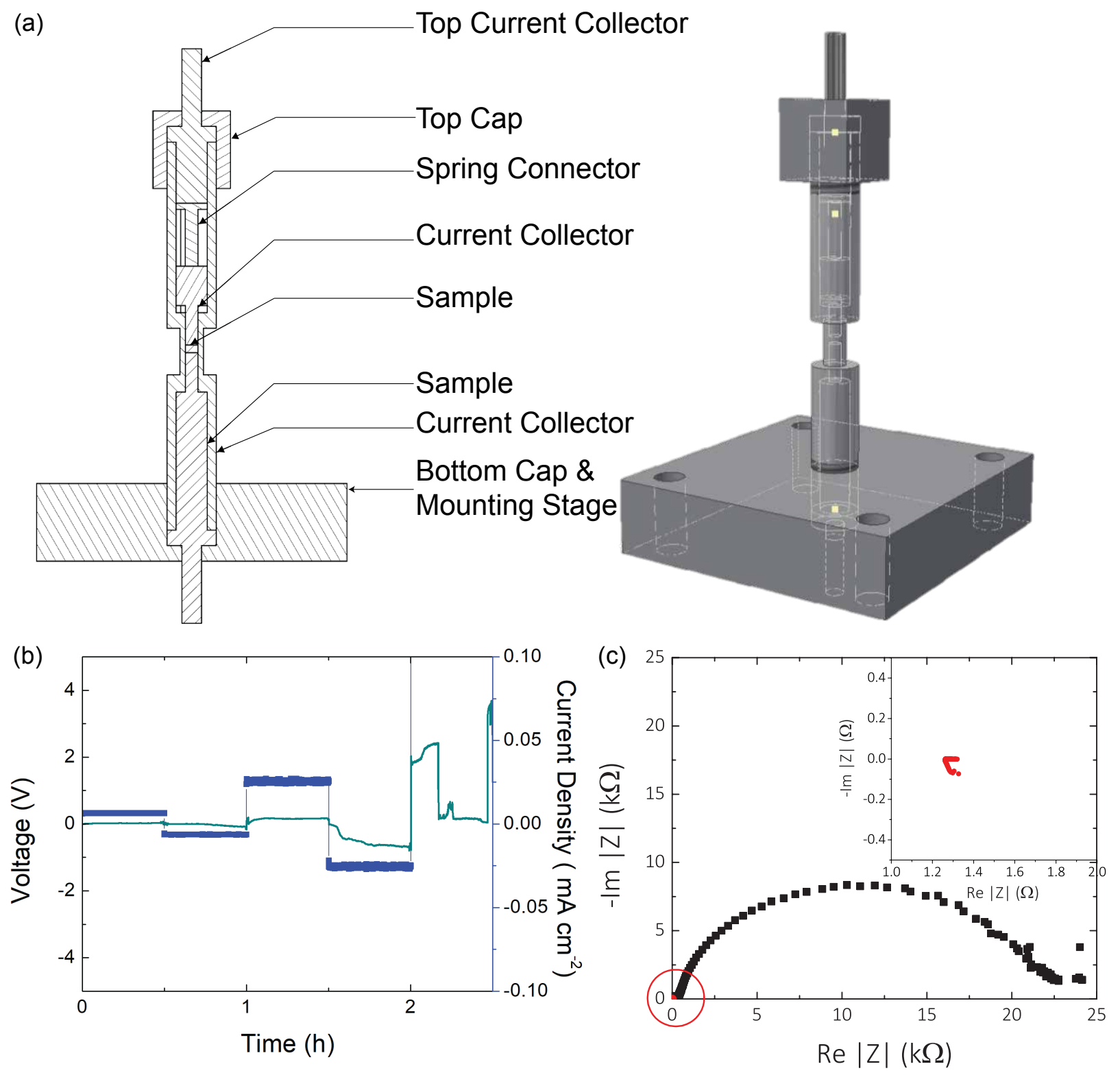

Fig S 3: In situ electrochemical measurements. (a) Schematic diagram showing the construction of an air sensitive cell for carrying out in situ experiments at the beamline. (b) Polarization profiles for plating and stripping measured on Li | LLZO | Li cells. A total of $1.5 \mathrm{mAh} \mathrm{cm}^{-2}$ lithium is cycled before failure. (c) Impedance measurements of the pristine and the failed cell(inset). 

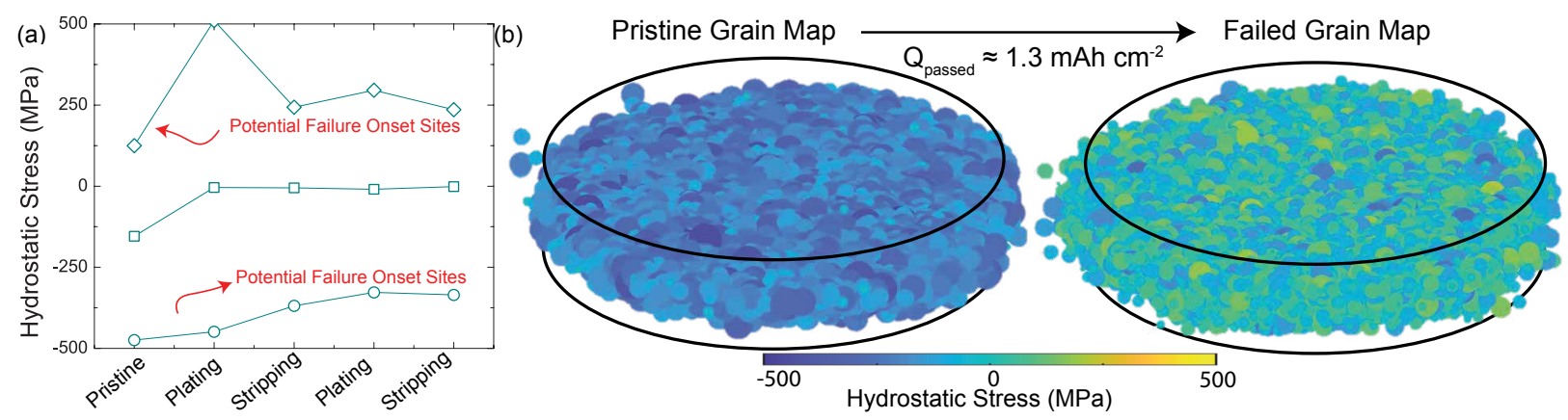

Fig S 4: Strain Response of Poly-crystalline LLZO Material for additional sample. (a)Hydrostatic stress evolution during cycling of Li|LLZO|Li sample. The stressvalues are averaged over all the grains in the measured sample. Maximum and minimum values are shown for individual grains in the sample. (d) 3D grain maps for pristine and failed sample with the grains color mapped to the stress values. A total of $1.3 \mathrm{mAh} \mathrm{cm}{ }^{-2}$ lithium is cycled before failure.
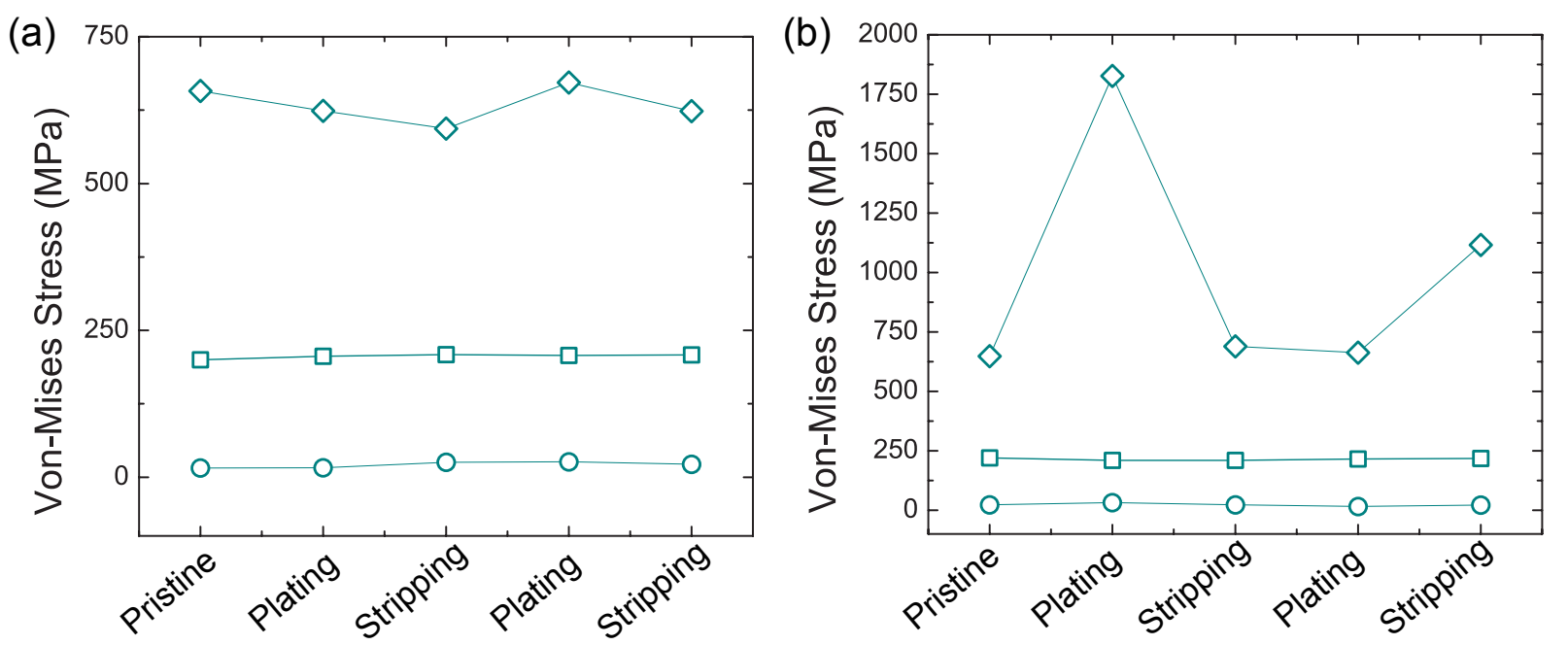

Fig S 5: Stress Response of Poly-crystalline LLZO Material. Von-Mises stress mapped over multiple electrochemical cycling for two samples measured (a-b).

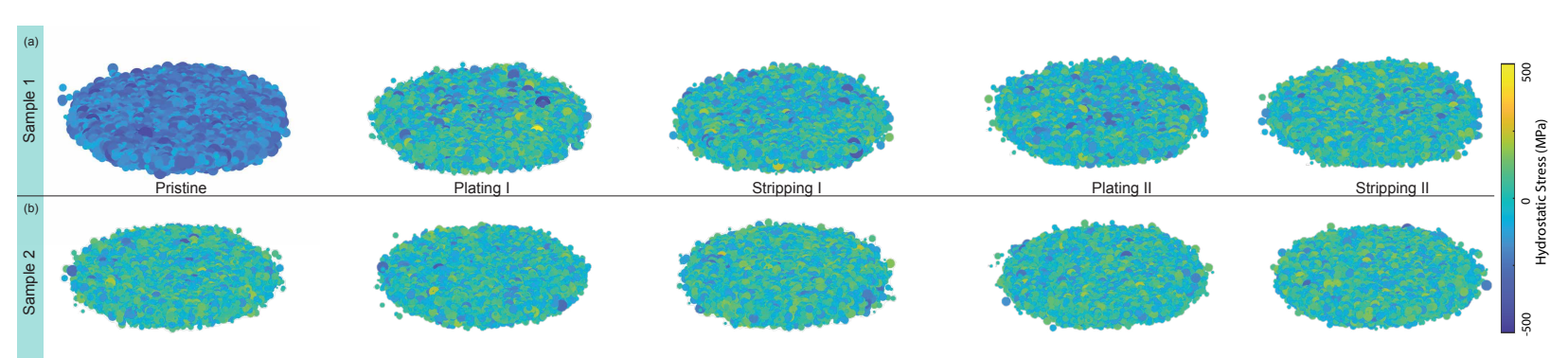

Fig S 6: Grain Maps for all the steps for two samples (a-b). The color mapping represents the hydrostatic stress for individual grains. 

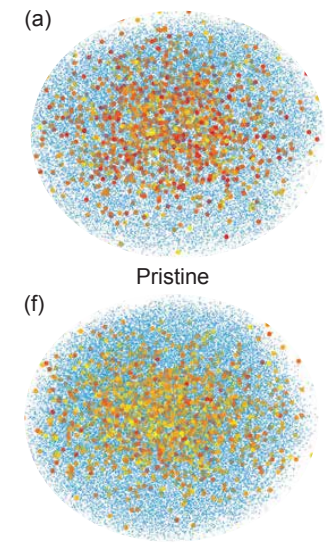

(b)

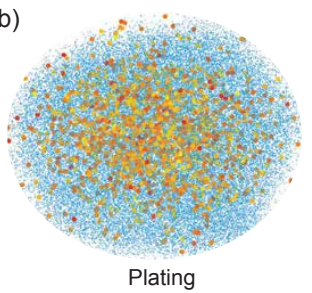

(g)

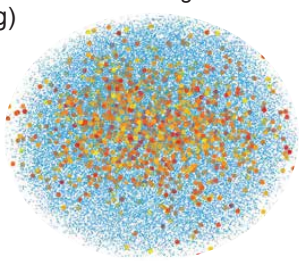

(c)

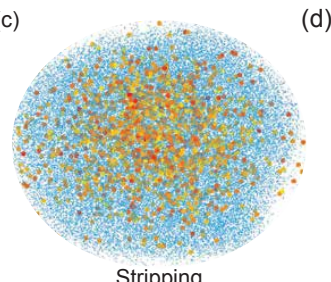

(h)

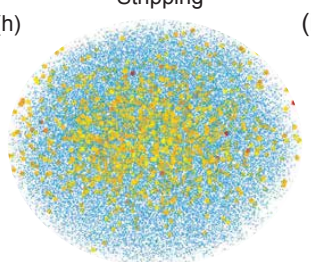

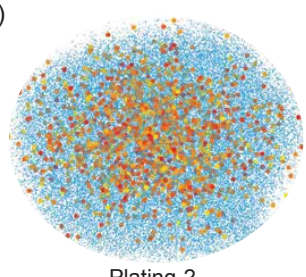

(i)

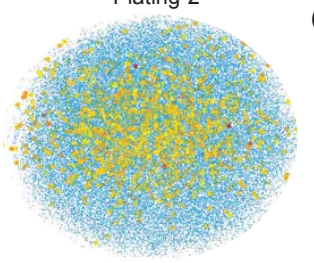

(e)
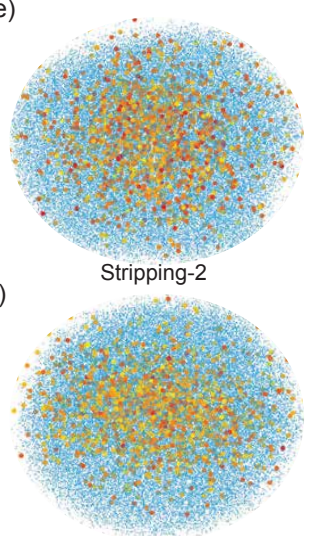

Fig S 7: Superposition maps for 220 SG grains (light blue) and 230 SG grains (larger scatter points, color mapped to stress state) for sample 1 (a-e) and sample 2 (f-j) at various states of electrochemical cycling. 

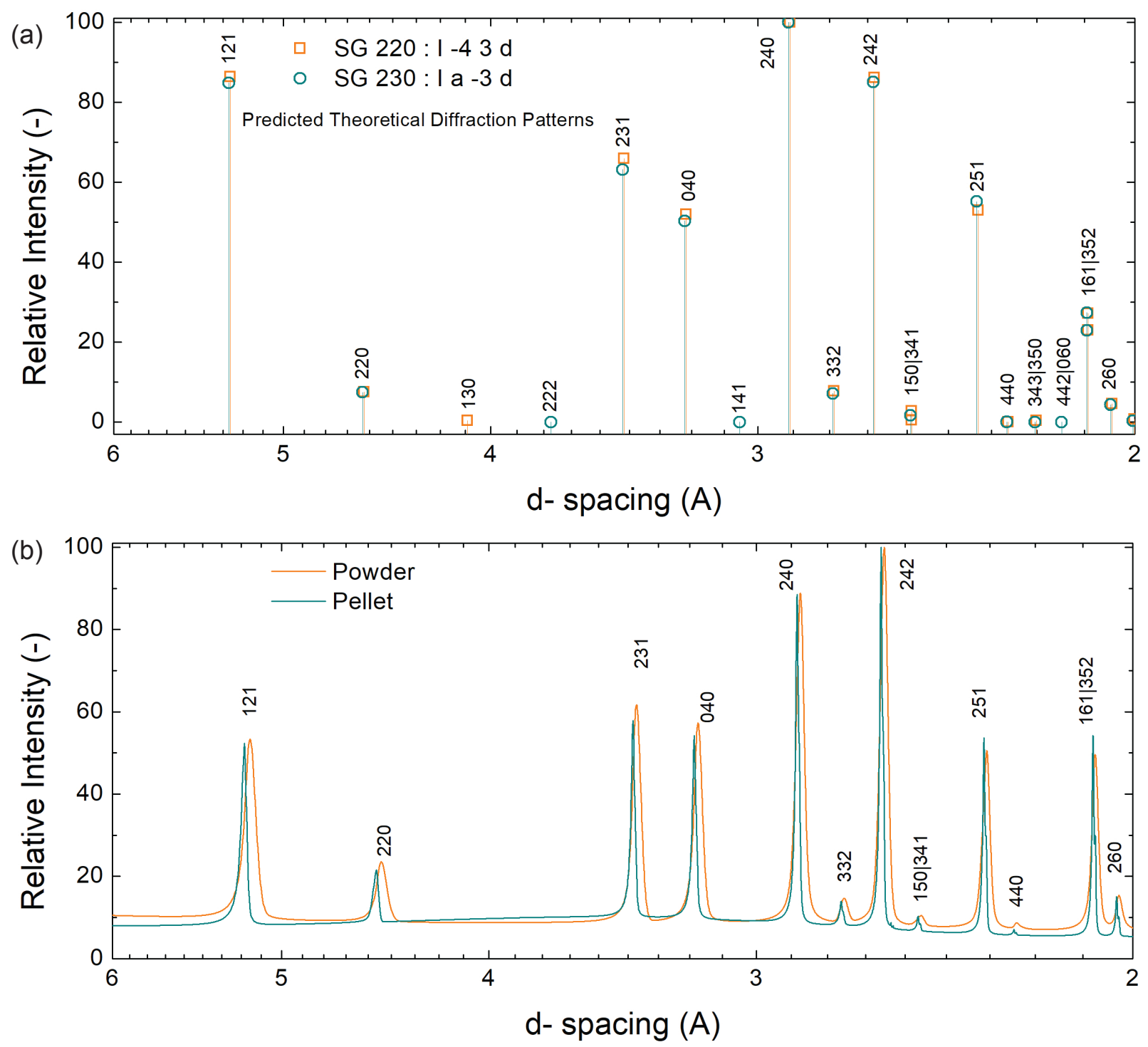

Fig S 8: Theoretical and laboratory XRD patterns for LLZO. (a) Theoretical XRD patterns for 220 and $230 \mathrm{sg}$. LLZO with corresponding $<\mathrm{hkl}>$ values mentioned. The only distinct reflections between the 220 and $230 \mathrm{sg}$. have low structure factors. (b) Laboratory XRD patterns of LLZO powder and pellet material. No evidence of the low structure factor reflections are observed in these patterns. 


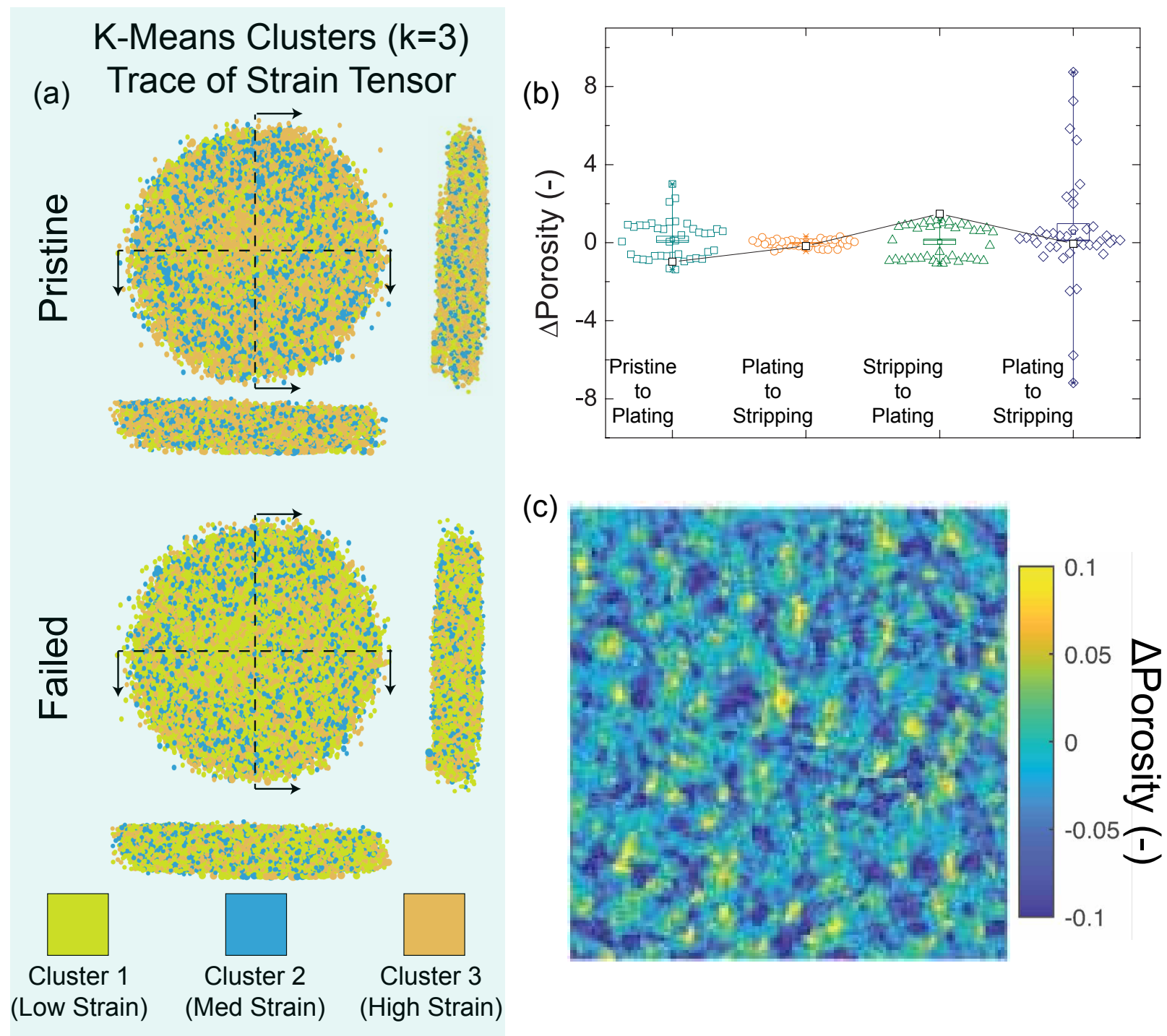

Fig S 9: Hydrostatic stress and microstructure evolution during cycling for additional sample. (a) Full pellet assessment for direction stress behavior using unsupervised machine learning algorithm, K-means. No preferential spatial distribution for the hydrostatic stress for individual grains is observed between the pristine and the failed sample. (b) Difference in porosity values tracked over the hot-spot and cold-spots for each step plotted as a box plot. Additionally, the average porosity difference over the entire pellet between each step is overlayed and connected by a solid line. As the sample undergoes degradation with cycling, the hot-/cold- neighborhoods clearly show very high variance in the porosity change compared to the bulk sample value. (c) Depth averaged $\Delta$ porosity values for identical sub-volume sizes taken at a hot spot region (right). The porosity difference is calculated between the pristine and the failed sample. The scale bar in the figure is $10 \mu \mathrm{m}$. 


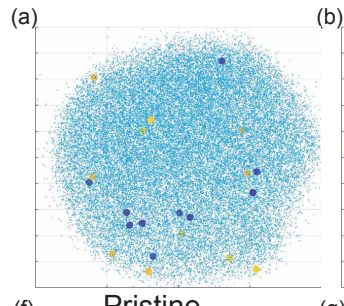

(f)

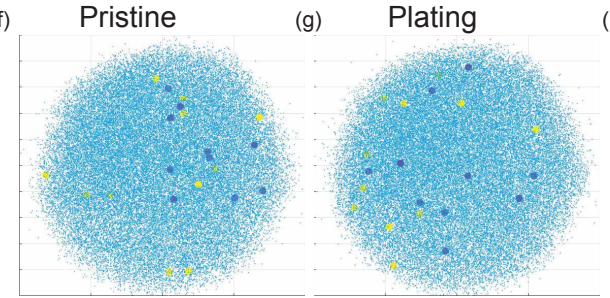

(c)

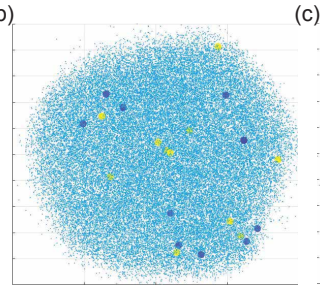

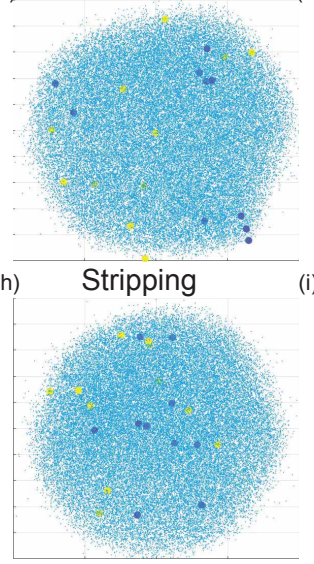

(d)
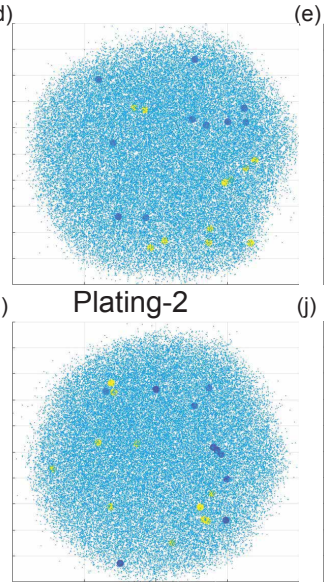

(e)

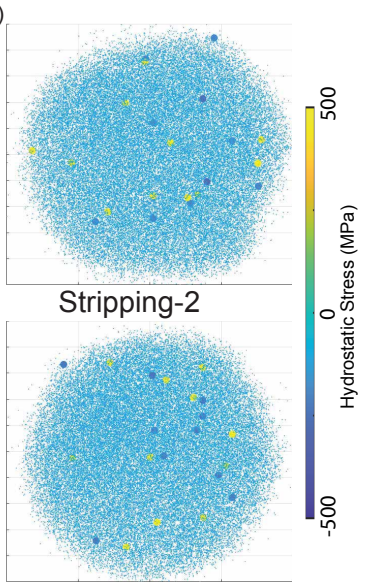

Fig S 10: Identification of hot-/cold- spots for LLZO across various stages of electrochemical cycling (a-e) for sample 1 and (f-j) for sample 2. Hot- and cold- spots are defined as ten grains with the highest and lowest hydrostatic stress in the measured pellet grain map and are depicted with larger scatter sizes. The color for the rest of the grains is merely for representation and does not signify any physical quantity. 
Tracking High Strain Neighborhoods During Cycling Tracking Low Strain Neighborhoods During Cycling

(a)

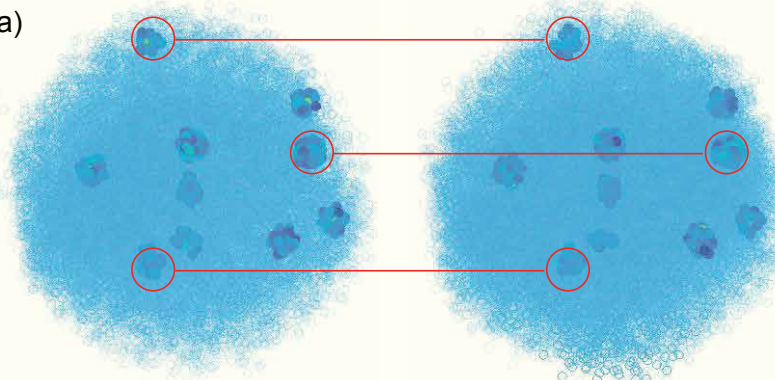

Plating

(c)

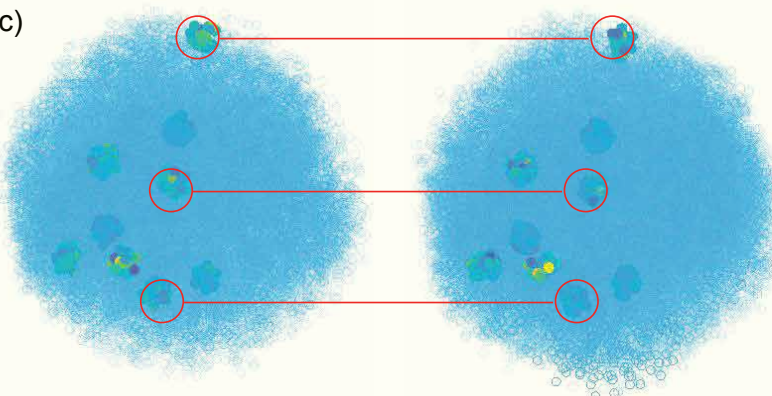

Stripping

(e)

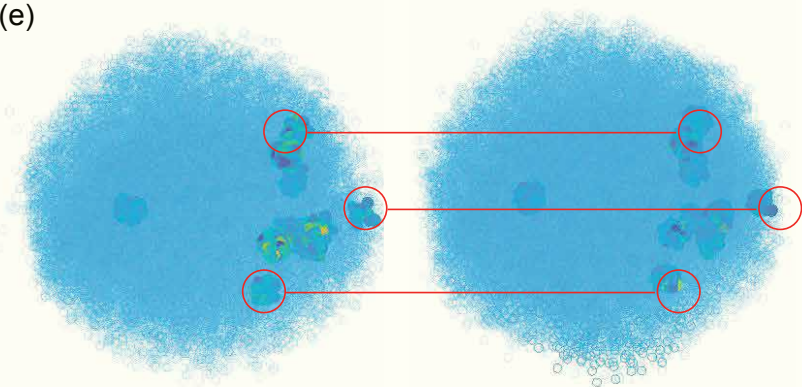

Plating-2
Stripping

Plating-2

Stripping-2 (b)

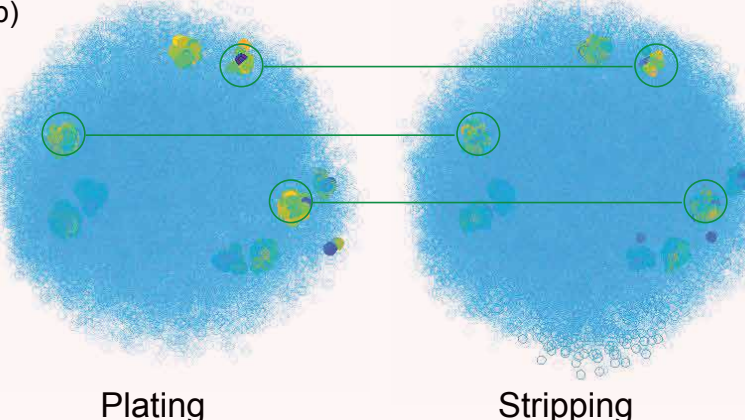

(d)

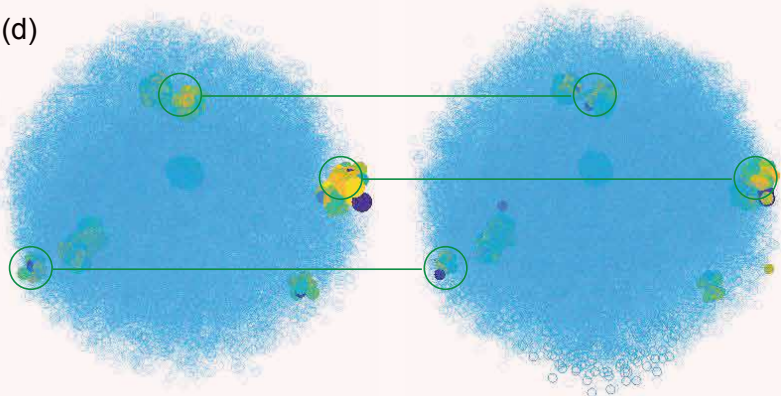

Stripping Plating-2

(f)

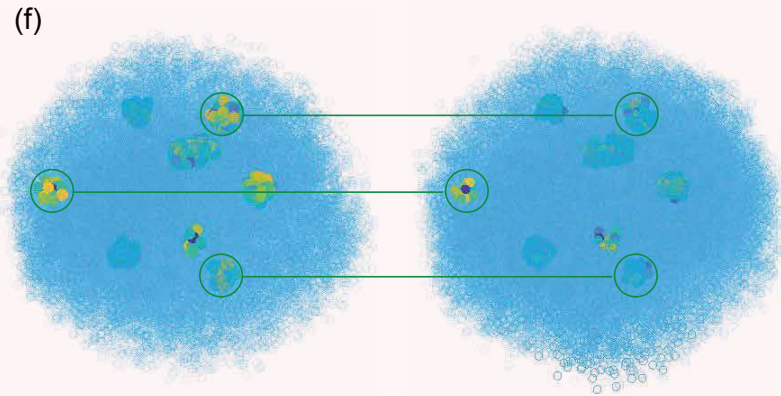

Plating-2

Stripping-2

Fig S 11: Hydrostatic stress evolution during cycling. Tracking of high stress and low stress neighborhoods at different stages of cycling for sample 1. (a-b) Plating to stripping. (c-d) Stripping to Plating-2. (e-f) Plating-2 to Stripping-2. 
(a)

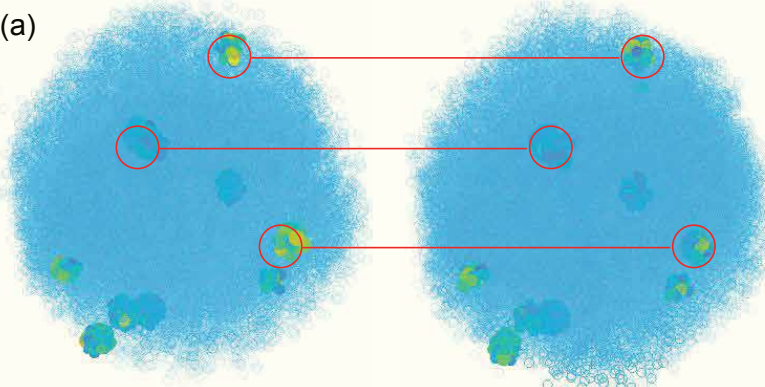

Pristine

(c)

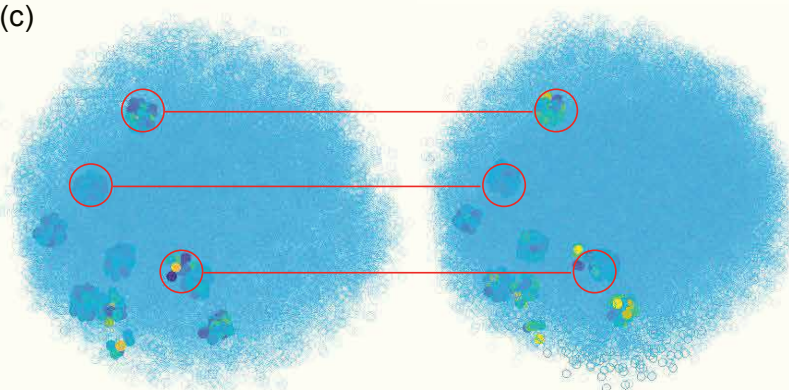

Plating

(e)

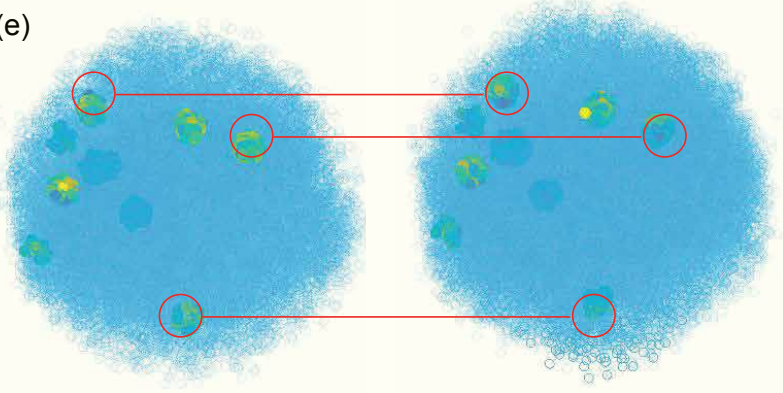

Stripping

(g)

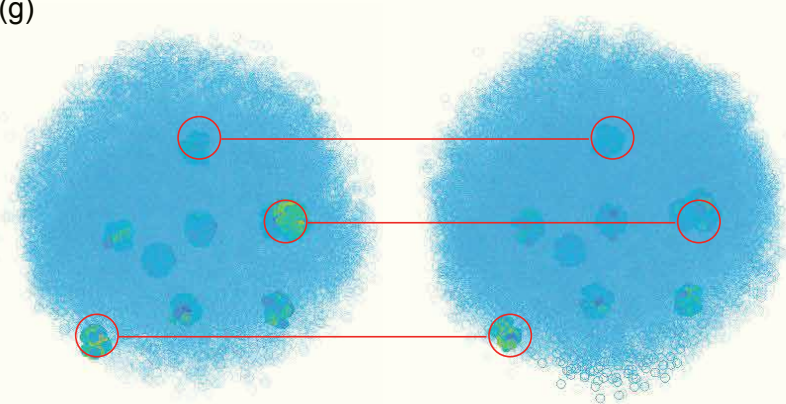

Plating-2

Stripping-2

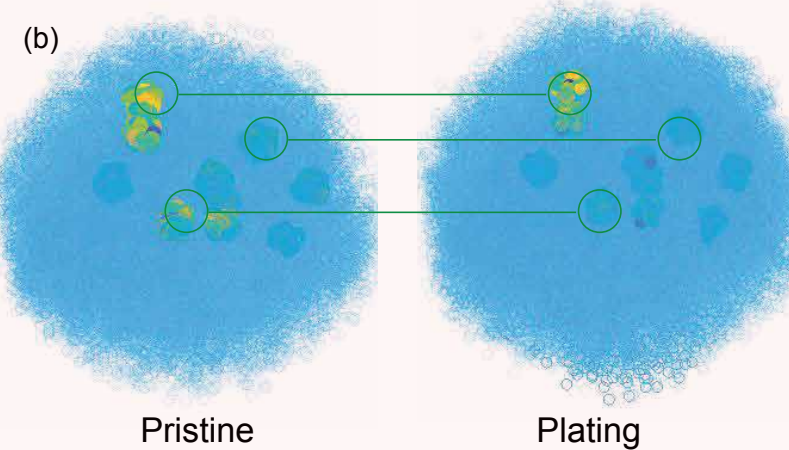

(d)

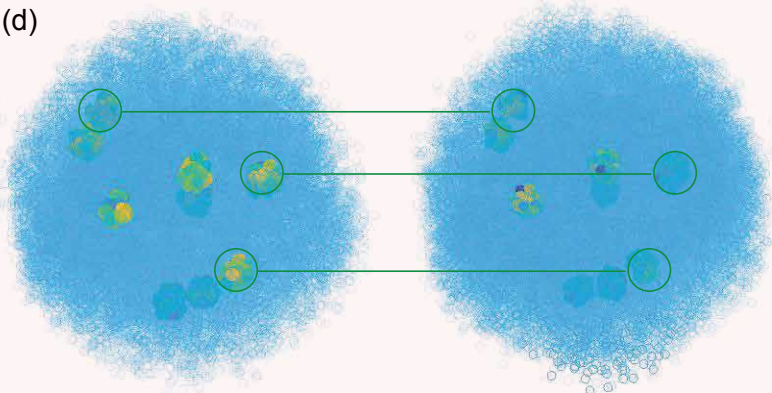

Plating

Stripping

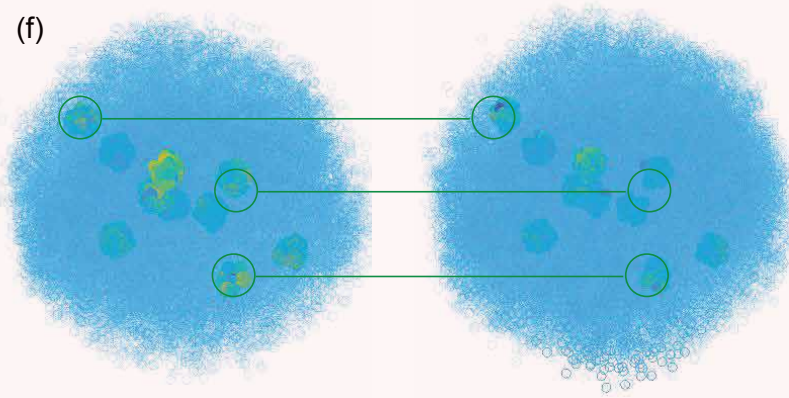

Plating-2

(h)

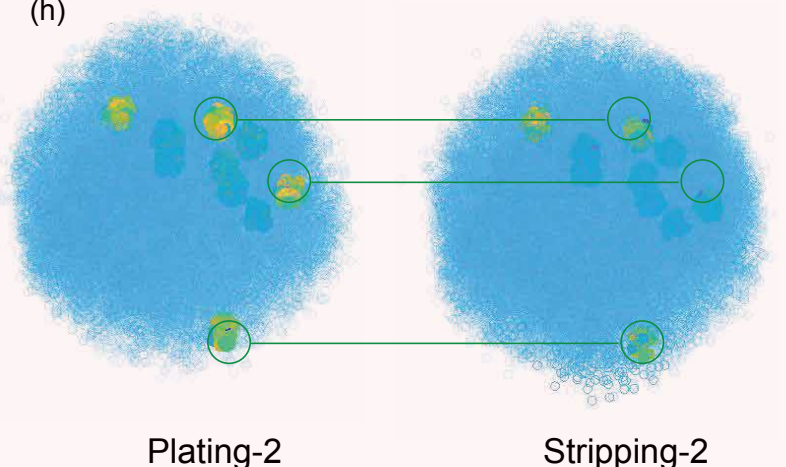

Fig S 12: Hydrostatic stress evolution during cycling. Tracking of high stress and low stress neighborhoods at different stages of cycling for sample 2. (a-b) Pristine to plating. (c-d) Plating to stripping. (e-f) Stripping to Plating-2. (g-h) Plating-2 to Stripping-2. 

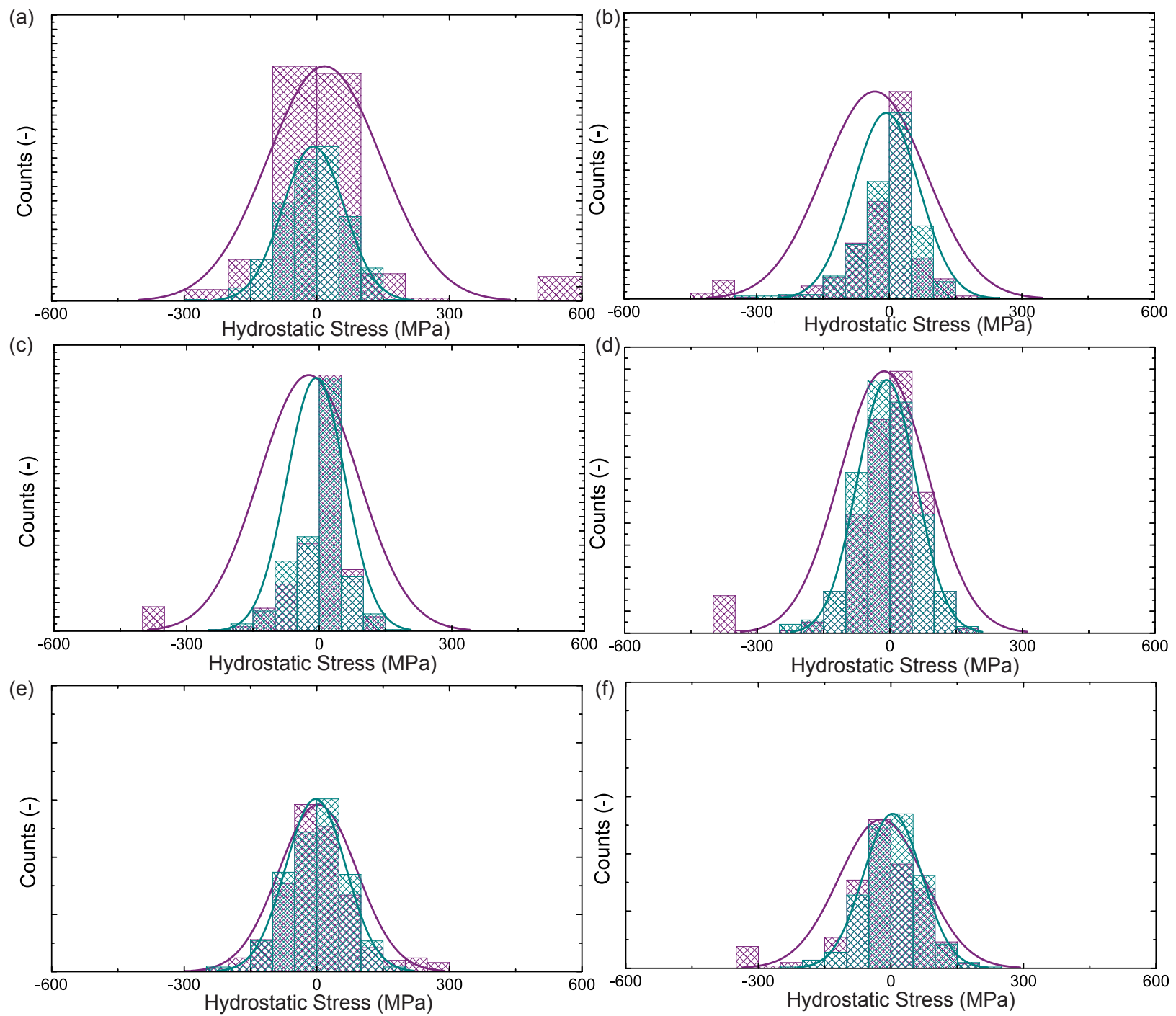

Fig S 13: Hydrostatic stress evolution during cycling. Tracking of high stress and low stress neighborhoods at different stages of cycling for sample 1 with the corresponding histograms and normal distribution fitting for the hydrostatic stress value for the grains in the identified neighborhood. (a-b) Plating to stripping. (c-d) Stripping to Plating-2. (e-f) Plating-2 to Stripping-2. 

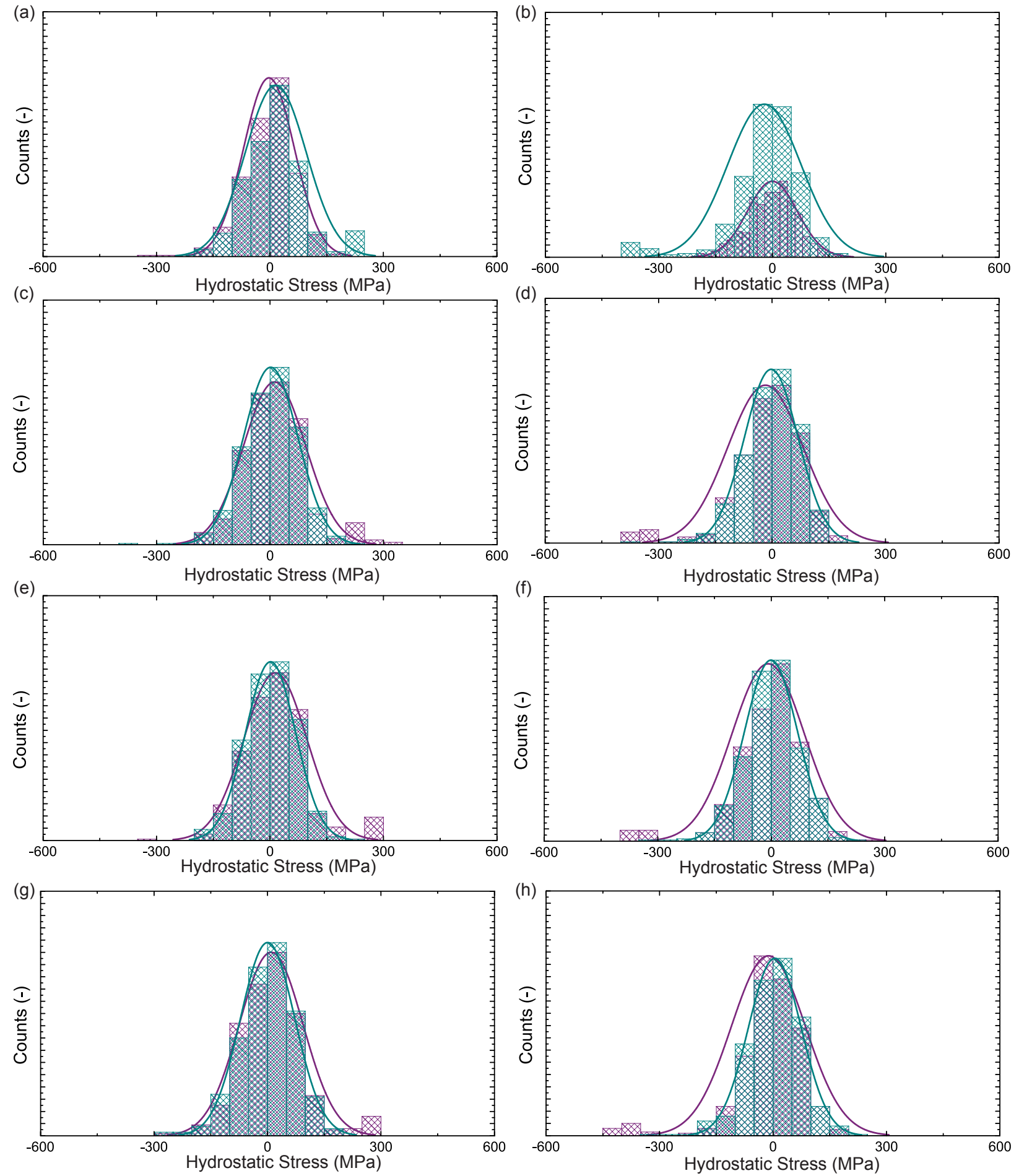

Fig S 14: Hydrostatic stress evolution during cycling. Tracking of high stress and low stress neighborhoods at different stages of cycling for sample 2 with the corresponding histograms and normal distribution fitting for the hydrostatic stress value for the grains in the identified neighborhood. (a-b) Pristine to plating. (c-d) Plating to stripping. (e-f) Stripping to Plating-2. (g-h) Plating-2 to Stripping-2. 

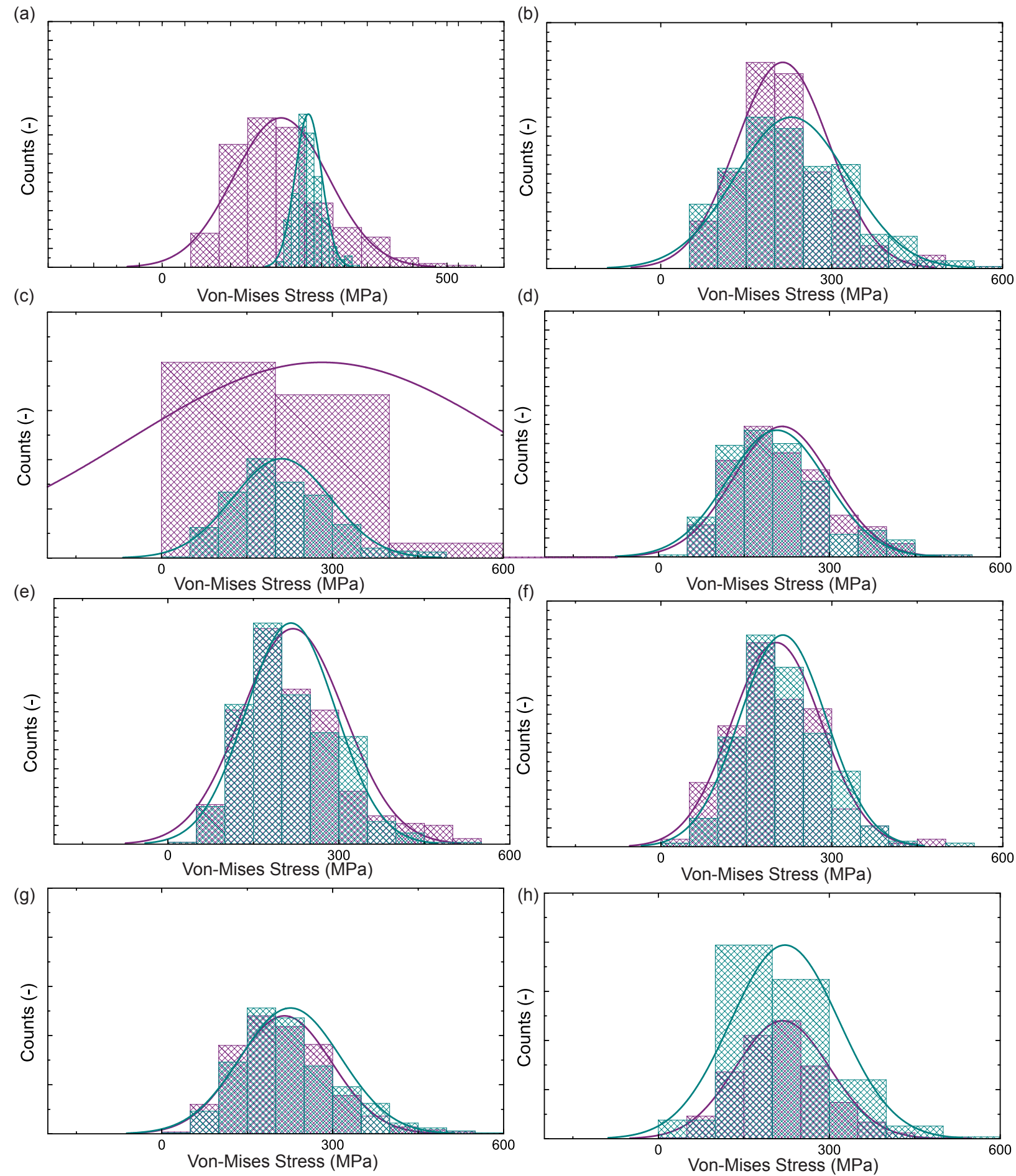

Fig S 15: Von-Mises stress evolution during cycling. Tracking of high stress and low stress neighborhoods at different stages of cycling for sample 1 with the corresponding histograms and normal distribution fitting for the hydrostatic stress value for the grains in the identified neighborhood. (a-b) Pristine to plating. (c-d) Plating to stripping. (e-f) Stripping to Plating-2. (g-h) Plating-2 to Stripping-2. 

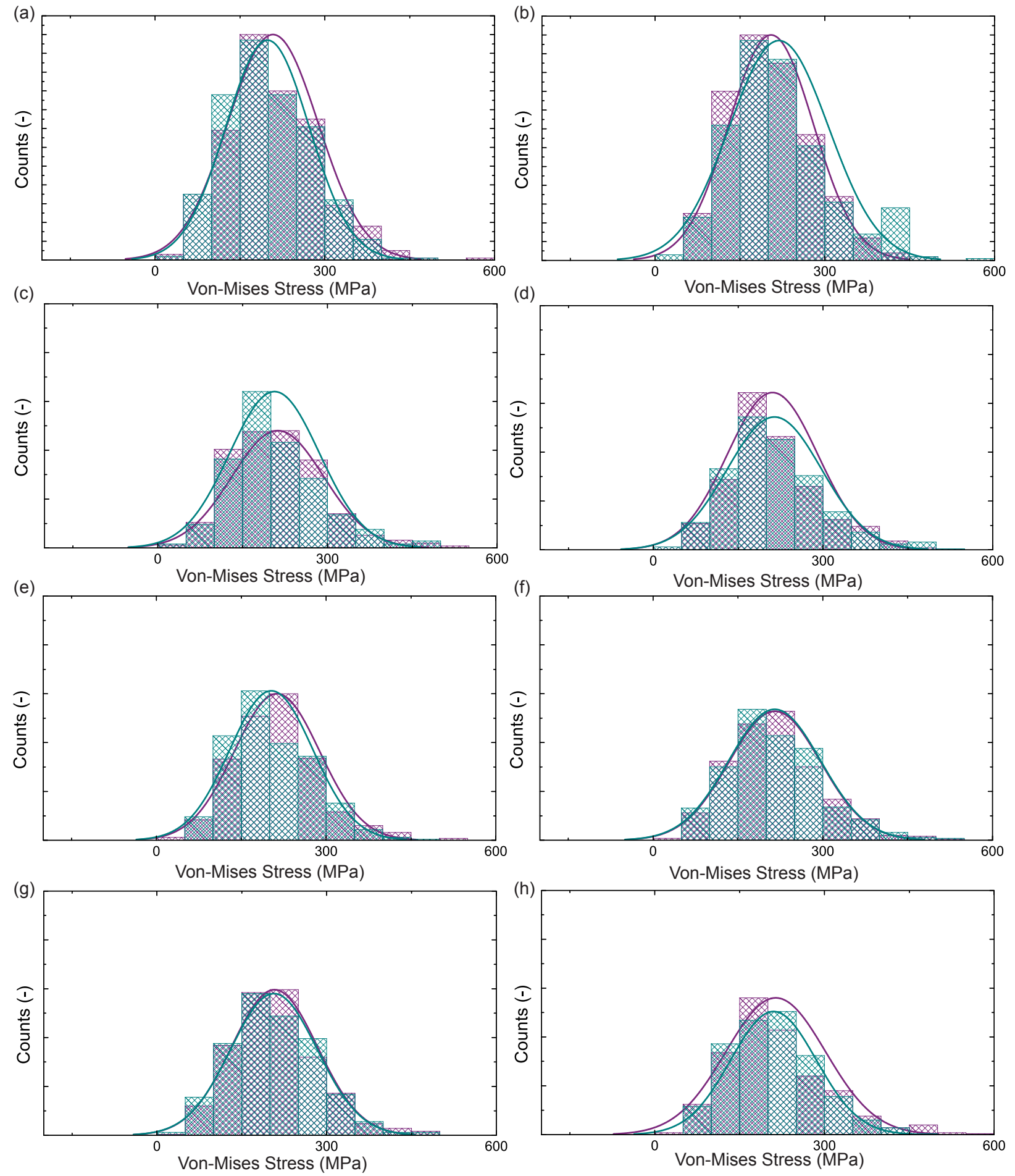

Fig S 16: Von-Mises stress evolution during cycling. Tracking of high stress and low stress neighborhoods at different stages of cycling for sample 2 with the corresponding histograms and normal distribution fitting for the hydrostatic stress value for the grains in the identified neighborhood. (a-b) Pristine to plating. (c-d) Plating to stripping. (e-f) Stripping to Plating-2. (g-h) Plating-2 to Stripping-2. 


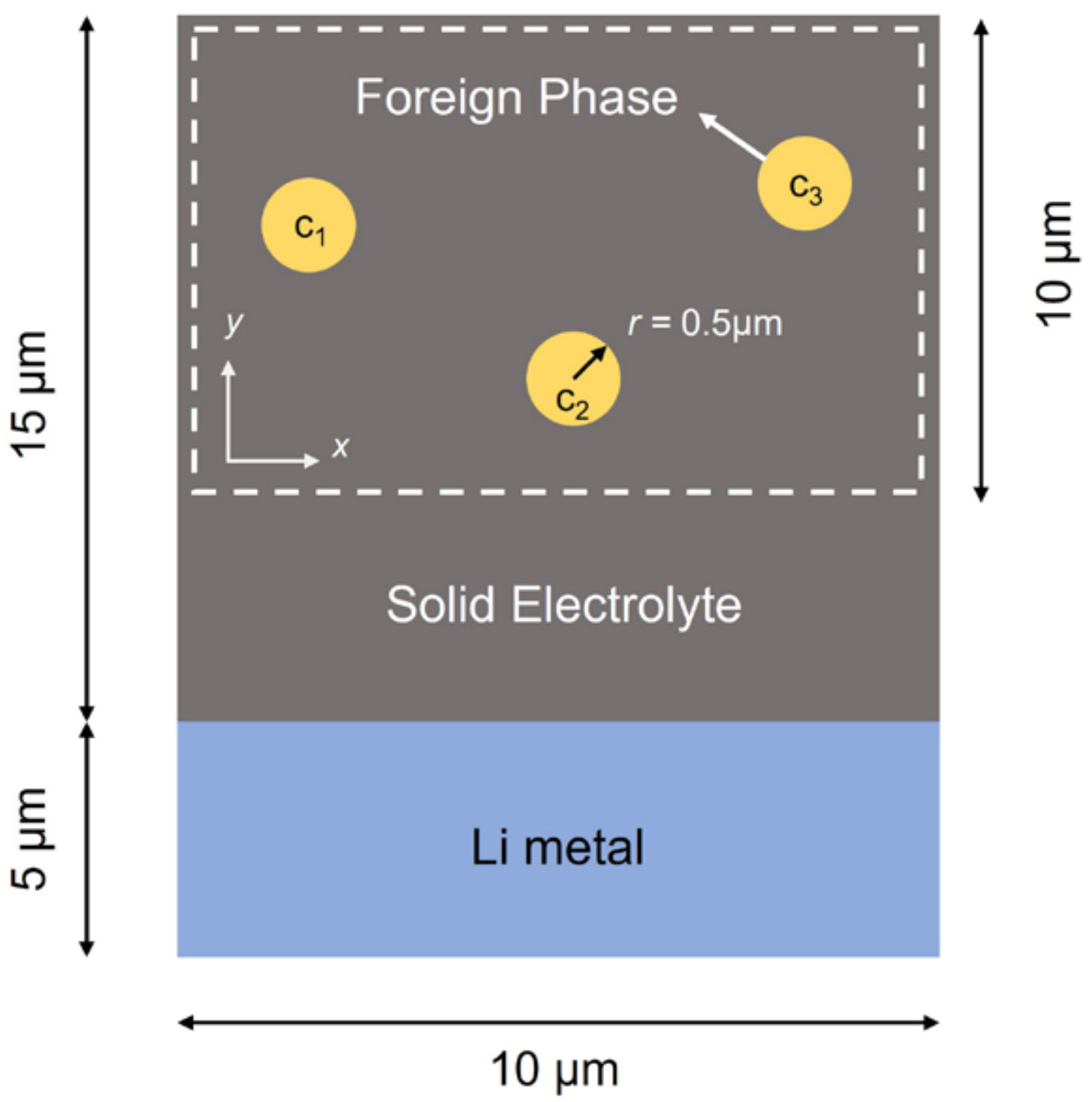

Fig S 17: Schematic diagram showcasing the simulation domain employed for the meso-scale modeling of electrochemical and mechanical response of LLZO with a secondary phase. 


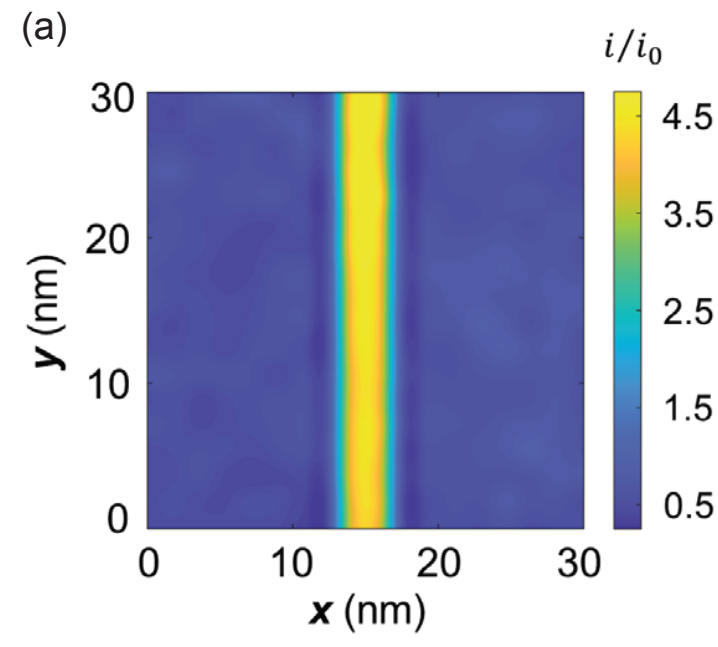

Local Li+ Occupation

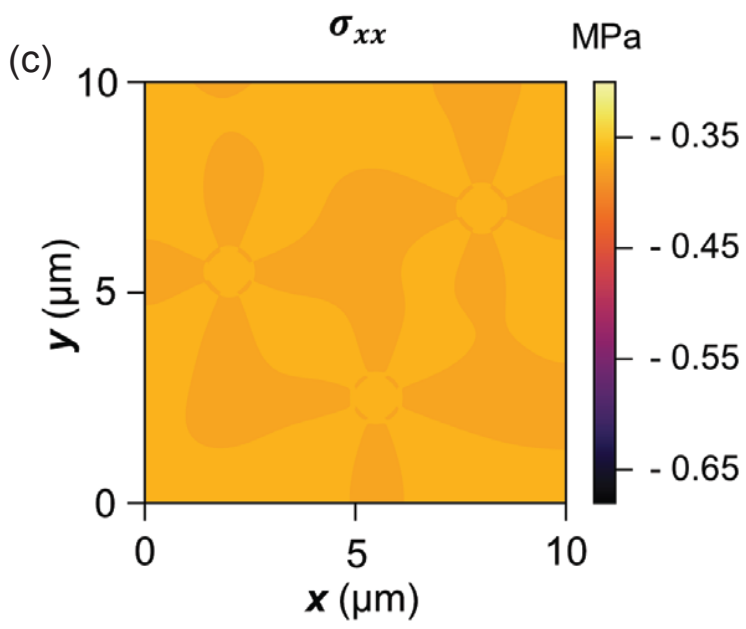

Mechanical Occupation

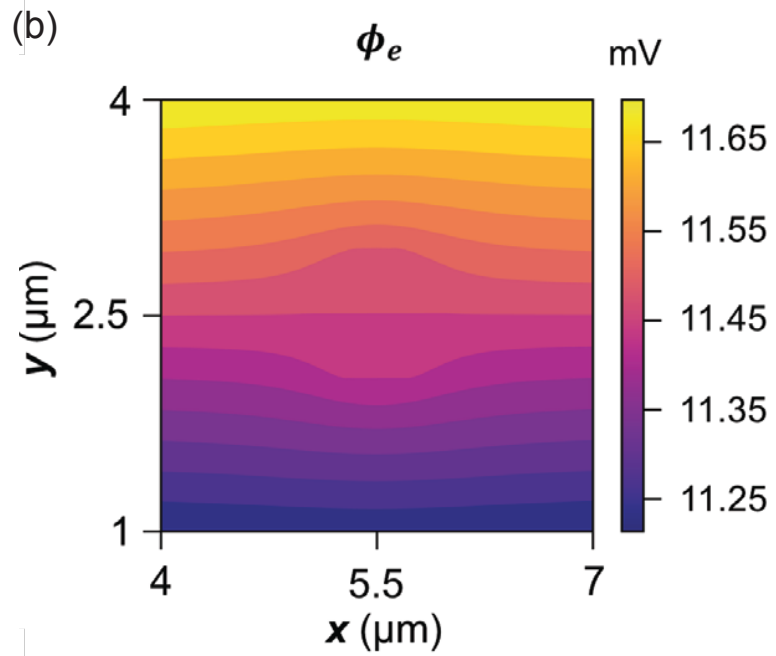

Field Response

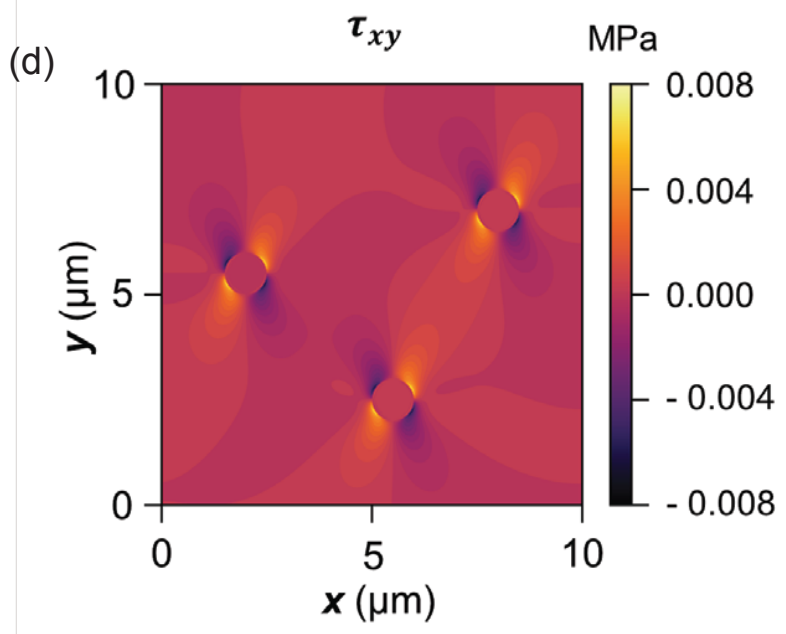

Mechanical Response

Fig S 18: Additional Modeling Results. (a) Mesoscale modeling results highlighting the variation in local Li occupation due to presence of the secondary phase, with a differing ion migration barrier when compared to the bulk. (b) Electric potential and (c-d) Mechanical response of a domain with embedded secondary phases. For the local occupation and field response, the secondary phase is considered more conductive than the primary domain. 

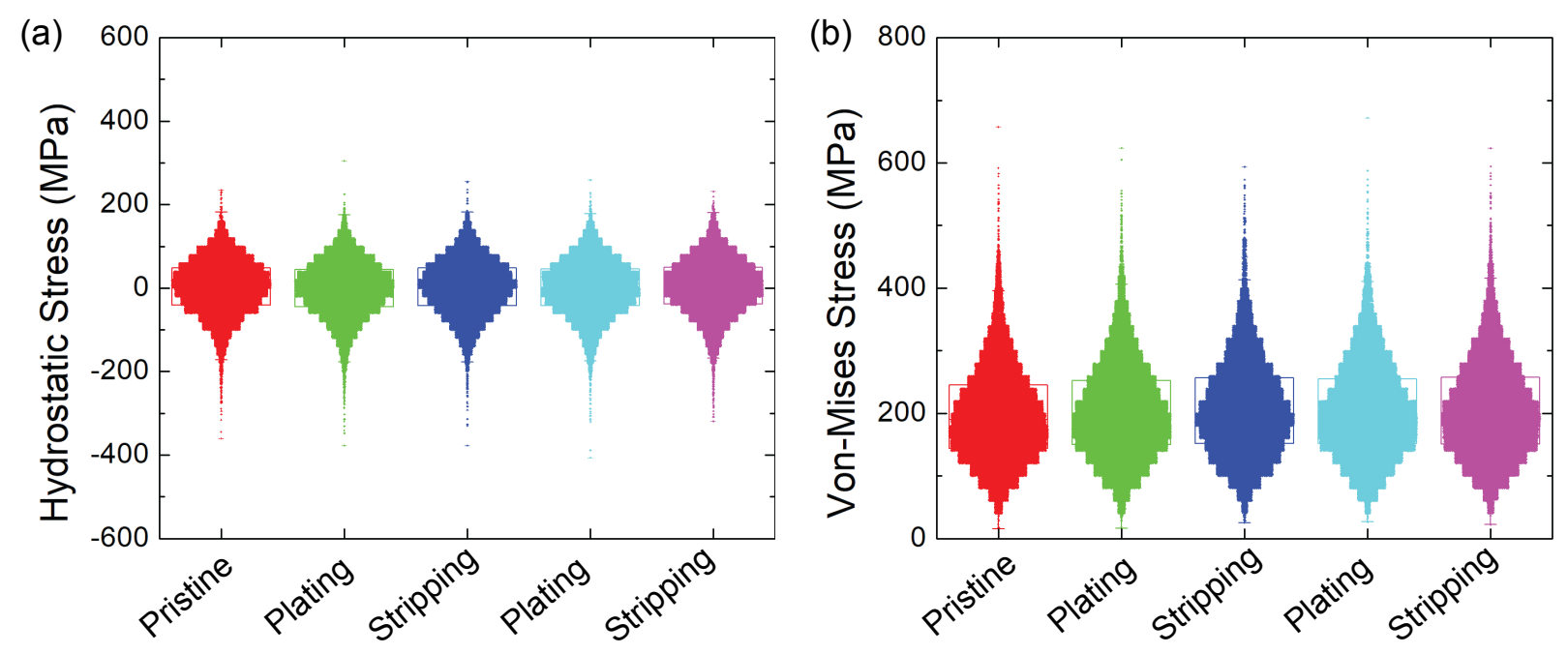

Fig S 19: Stress Response of Poly-crystalline LLZO Material. Violin plots depicting the overall stress distribution along with the individual data sets for hydrostatic (a,c) stress and Von-Mises stress $(b, d)$ for additional sample. The width of the violin plots indicate the number density of grains at the specified stress value.
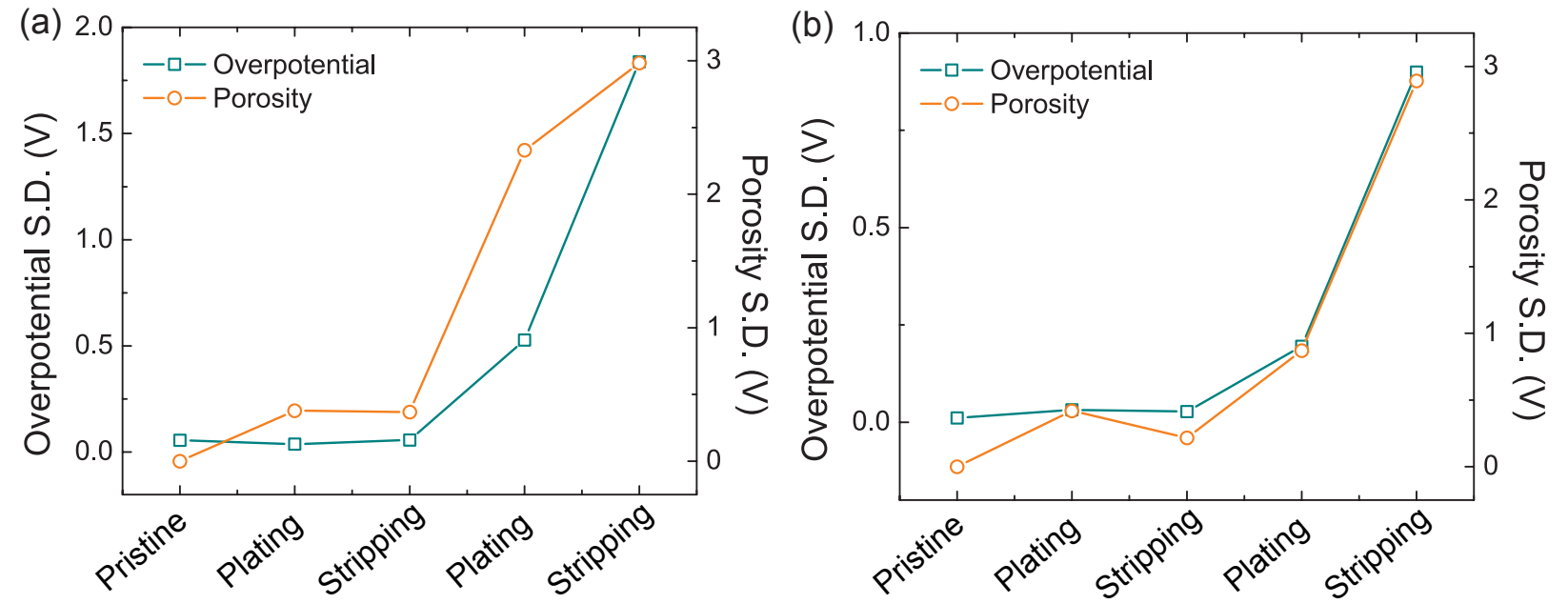

Fig S 20: Correlation between cell degradation and the local change in porosity. Standard deviation for the observed Li plating/stripping overpotential during each half-cycle and the standard deviation of the local porosity of the hot-spots identified are plotted for (a) Sample 1 and (b) Sample 2. Strong correlation between the two data-sets are observed. 


\section{References}

[1] Marm B Dixit, Ankit Verma, Wahid Zaman, Xinlin Zhong, Peter Kenesei, Jun Sang Park, Jonathan Almer, Partha P Mukherjee, and Kelsey B Hatzell. (2020). Synchrotron Imaging of Pore Formation in Li Metal Solid-State Batteries Aided by Machine Learning. ACS Applied Energy Materials. 3(10):9534-9542. 